\title{
ABHANDLUNG
}

\section{Ursachen kommunaler Haushaltsdefizite}

\author{
Jörg Bogumil / Lars Holtkamp / Martin Junkernheinrich / Uwe Wagschal
}

\section{Determinants of local budget deficits}

Abstract: This contribution investigates the exogenous and endogenous determinants of local government debts in Germany. The municipality cannot influence exogenous factors, whereas endogenous factors are largely driven by the local political competition and exhibit their effects mainly in the local context. Triangulation of different methods is used to reach robust results, encompassing both quantitative research methods and qualitative methods like case studies and interviews. The analysis can thus show, that the local debts are not only explained by socioeconomic, demographic, and income factors, but that political-institutional factors like the local constitutional law, the mayor's party affiliation and the size of the local council are also relevant. In addition, the case studies lend support to the hypothesis that consensus arrangements in the municipality lead to a better financial situation.

Keywords: Local politics, public debt, local constitution

Schlagwörter: Kommunalpolitik, Verschuldung, Kommunalverfassung

\section{Einleitung}

Seit vielen Jahren befindet sich eine Vielzahl von Kommunen in Deutschland in einer tiefgreifenden Haushaltskrise. Allerdings sind nicht alle Kommunen gleichermaßen von der Haushaltskrise betroffen. Hinter den aggregierten Schuldendaten verbergen sich auf Länder-, Gemeindeverbands- und Gemeindeebene erhebliche Disparitäten. Einigen Kommunen gelingt es auch in Zeiten ungünstiger wirtschaftlicher Entwicklung, ihre Etats ohne Neuverschuldung auszugleichen. Es besteht also trotz der im Durchschnitt steigenden Verschuldung eine erhebliche Varianz der Haushaltssituation der Kommunen in Deutschland. Vor diesem Hintergrund wird in diesem Papier auf der Datengrundlage eines DFG-Gemeinschaftsprojektes nach den Ursachen kommunaler Haushaltsdefizite gefragt. ${ }^{1}$ Die

1 Die hier vorgelegten Ergebnisse basieren auf einem gemeinsamen DFG-Forschungsprojekt der vier Autoren, in dem sowohl die Haushaltsdefizite auf der Makro- (alle Kommunen über 5000 Einwohner), der Meso- (alle Kommunen über 10.000 Einwohner) und der Mikroebene (es wurde 18 vertiefende Fallstudien mit insgesamt 75 Experteninterviews in vier Bundesländer durchgeführt) für den Zeitraum 2003-2010 untersucht wurden. Wissenschaftliche Mitarbeiter in diesem Projekt waren zu unterschiedlichen Zeiträumen Thomas Bathge, Florian Boettcher, David Boll, Falk Ebinger, Dominik Frankenberg, Tobias Fuhrmann, Maximilian Grasl, Benjamin Holler, Hendrik Markert und Marc Seuberlich (vgl. auch Boettcher 2013; Holtkamp u. Bathge 2012a, 2012b; Wagschal u. Grasl 2014; Seuberlich 2012, 2013). 
leitende Fragestellung ist dabei: Welchen Einfluss haben politische, sozioökonomische und institutionelle Faktoren und Rahmenbedingungen sowie Interaktionsmuster und Handlungsorientierungen kommunaler Akteure auf die kommunale Haushaltssituation?

Die Ursachen für die unterschiedliche Leistungsfähigkeit der Kommunen können in exogene und endogene Faktoren unterschieden werden. Exogene Faktoren sind solche Einflussvariablen, die nicht von den Kommunen selbst beeinflusst werden können und zumindest kurz- und mittelfristig konstant sind, wie etwa die Fluktuation des Wirtschaftswachstums, die Höhe der Arbeitslosenquote oder auch der Anteil der Sozialhilfeempfänger. Solche sozioökonomischen und institutionellen Gründe führen dazu, dass die Haushaltsprobleme der Kommunen aus dieser Sicht kaum selbst zu verantworten sind und sich die Kommunen als „Gefangene“ oder „Opfer“ („Opferthese“) bezeichnen (Holtkamp 2010). Vor dem Hintergrund der unterschiedlichen Theorien der Staatstätigkeitsforschung (Schmidt 1993, 2001) können weitere verschiedene exogene Faktoren identifiziert werden, die generell der sozioökonomischen, internationalen oder politischinstitutionellen Theorieschule zuzurechnen sind. Hinzu kommen Variablen, die die Pfadabhängigkeit messen, ein Ansatz der in der Policy-Forschung ebenfalls verbreitet ist (Rose u. Karran 1987).

$\mathrm{Zu}$ den endogenen Faktoren sind solche Variablen zu rechnen, die aus dem politischen Wettbewerb heraus die Verschuldungssituation erklären können. Diese „selbstgemachten“ Probleme bzw. Faktoren sind daher auf der kommunalpolitischen Ebene verordnet und werden typischerweise mit Variablen wie der Parteizugehörigkeit des Oberbürgermeisters, den unterschiedlichen Stärkeverhältnissen oder auch der parteipolitischen Zersplitterung der politischen Kräfte in der Kommunalvertretung erklärt (als Indikator für den politischen Wettbewerb). Die Erklärungskraft endogener und exogener Einflussfaktoren auf die Finanzlage sollte daher variieren. Durch die hohe Verflechtungsintensität der Kommunen untereinander sowie mit den übergeordneten Ebenen ist auszuschließen, dass die Finanzlage ausschließlich endogen oder exogen determiniert wird (Timm-Arnold 2011, S. 445-446).

Um die Fragen nach den Determinanten der Kommunalverschuldung beantworten zu können, wird zunächst im zweiten Abschnitt die Verschuldungssituation der Kommunen dargestellt und es werden unterschiedliche abhängige Variablen diskutiert. Im dritten Abschnitt werden dann die Hypothesen für den empirischen Test aus unterschiedlichen Theorien der Staatstätigkeitsforschung beziehungsweise der politischen Ökonomie abgeleitet. Abschnitt vier präsentiert die Befunde der quantitativen Analyse. Der methodische Zugang ist jedoch nicht nur quantitativ, sondern wird - im Sinne einer Triangulation (Flick 2008) - auch qualitativ gesucht (Abschnitt fünf). Das Kernanliegen dieses Beitrages ist die Anwendung unterschiedlicher sozialwissenschaftlicher Methoden zur Erforschung eines Untersuchungsgegenstandes, im vorliegenden Fall den Ursachen der Kommunalverschuldung, mit dem Hauptziel der Validierung der Forschungsergebnisse. 


\section{Die Verschuldungssituation der Kommunen im Überblick}

Die Staatsverschuldung als dauerhaftes Instrument der staatlichen Einnahmenerzielung etablierte sich insbesondere in den neunzehnhundertsiebziger Jahren auf allen staatlichen Ebenen der Bundesrepublik. Dabei gibt es zwischen den Gebietskörperschaften jedoch signifikante Unterschiede (Statistisches Bundesamt 2014a). Gemessen an dem Schuldenstand je Einwohner, bestand für den öffentlichen Gesamthaushalt (Bund, Länder, Sondervermögen des Bundes sowie Gemeinden und Zweckverbände) Ende 2013 in Deutschland eine durchschnittliche Pro-Kopf-Verschuldung von 25.289 Euro. Davon entfallen rund 62 Prozent auf den Bund (15.860 Euro pro Kopf), rund 31 Prozent auf die Länder (7755 Euro pro Kopf), etwa 7 Prozent auf die Kommunen (1807 Euro pro Kopf) und ein marginaler Teil auf die Sozialversicherungen (10 Euro pro Kopf).

Die Erfassung der exakten Höhe der Kommunalverschuldung ist jedoch weitaus komplexer und heterogener, als diese Daten suggerieren. Umstellungen in der statistischen Erfassung und Verbuchung sowie die Veränderungen in der Demografie (etwa durch den Zensus 2011) führten zu teilweise größeren Veränderungen der Daten. ${ }^{2}$ Hinzu kommt, dass nur die explizite Staatsverschuldung erfasst wird und nicht die implizite Staatsverschuldung. Letztere stellt ein Problem der exakten Erfassung der zukünftigen Lasten dar. So stehen die zukünftigen Pensionslasten, aber auch zukünftige Ausgaben, etwa für die Sanierungen der Infrastruktur, schon heute in hohem Maße fest, obwohl diese nicht verbucht werden.

2 Durch den Zensus von 2011 haben sich die ausgewiesenen Werte noch einmal in der Summe um etwa 500 Euro pro Kopf erhöht. Für den kommunalen Bereich macht dies etwa aktuell 30 Euro pro Kopf aus. Anfang 2014 hatten bundesweit 833 Kommunen Widerspruch gegen die vorgelegten Einwohnerzahlen eingelegt, 57 Kommunen hatten darüber hinaus Klage eingereicht (Bundestags-Drucksache 18/256). Der Sprung bei den Verschuldungsdaten im Jahr 2010 rührt von der Umstellung auf eine neue Erfassung der Verschuldungsdaten her, bei denen nun alle öffentlichen Fonds, Einrichtungen und Unternehmen des Staatssektors erfasst sind (Statistisches Bundesamt 2014a). 


\section{Abbildung 1: Das Schalenkonzept der öffentlichen Haushalte}

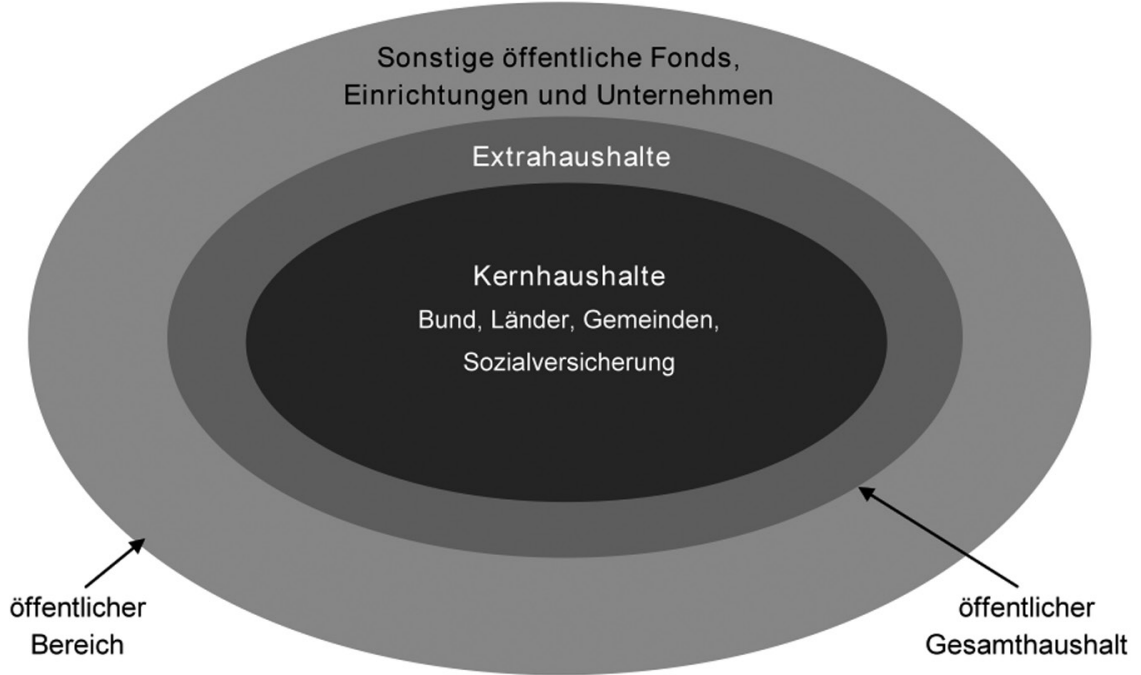

Quelle: Eigene Darstellung in Anlehnung an Statistisches Bundesamt 2014b

Hinzu kommt, dass die Haushalte auf kommunaler Ebene hoch fragmentiert sind, was an Auslagerungen in Extrahaushalten sowie der Schulden von öffentlichen Fonds, Einrichtungen und Unternehmen (FEU) liegt, die in unterschiedlichsten Rechtsformen (z. B. Eigenbetriebe, Zweckverbände) und Beteiligungsquoten geführt werden. ${ }^{3}$ Die Finanzstatistik reagierte auf diese Fragmentierung mit dem Aufbau eines konsolidierten Berichtssystems, dem sogenannten „Schalenkonzept der öffentlichen Aufgabenwahrnehmung “ (Abbildung 1). Es wurde im Jahre 2010 für die Schuldenstatistik implementiert und grenzt zwischen Kernhaushalten, Extrahaushalten sowie den sonstigen FEUs drei wesentliche Kategorien voneinander ab. Für die übrige Finanzberichterstattung erfolgte die Einführung ab 2011.

3 Eine integrierte Schuldenberichterstattung erfolgte erstmals mit dem „Schuldenreport“ der Bertelsmann Stiftung (Junkernheinrich u. Micosatt 2008). 
Tabelle 1: Kommunale Pro-Kopf-Verschuldung in den Bundesländern (inkl. Extrahaushalte, Zweckverbände, FEU) am 31.12.2012

\begin{tabular}{|c|c|c|c|c|c|c|}
\hline \multirow[t]{2}{*}{ Bundesland ${ }^{1}$} & \multirow{2}{*}{$\begin{array}{c}\text { Durchschnitt- } \\
\text { liche kommu- } \\
\text { nale Pro-Kopf- } \\
\text { Verschuldung } \\
\text { in Euro }{ }^{2}\end{array}$} & \multicolumn{3}{|c|}{$\begin{array}{l}\text { Anzahl der Städte und Gemein- } \\
\text { den mit kommunalen Schulden } \\
\text { je Einwohner... }\end{array}$} & \multirow{2}{*}{$\begin{array}{l}\text { Zahl der } \\
\text { Kommu- } \\
\text { nen im } \\
\text { Bundes- } \\
\text { land }\end{array}$} & \multirow{2}{*}{\begin{tabular}{|c|} 
Anteil der \\
hochver- \\
schuldeten \\
Kommu- \\
nen \\
$(>2000 €)$
\end{tabular}} \\
\hline & & \begin{tabular}{|c|} 
unter \\
500 Euro
\end{tabular} & $\begin{array}{l}\text { zwischen } \\
500 \text { und } \\
2000 \\
\text { Euro }\end{array}$ & $\begin{array}{l}\text { über } \\
2000 \\
\text { Euro }\end{array}$ & & \\
\hline Schleswig-Holstein & 2175 & 653 & 389 & 74 & 1116 & 6,6 \\
\hline Bayern & 2293 & 755 & 1072 & 225 & 2052 & 11,0 \\
\hline Sachsen & 2753 & 28 & 251 & 175 & 454 & 38,5 \\
\hline \begin{tabular}{|l|} 
Baden- \\
Württemberg \\
\end{tabular} & 2856 & 324 & 600 & 177 & 1101 & 16,1 \\
\hline Thüringen & 2901 & 62 & 490 & 326 & 878 & 37,1 \\
\hline Niedersachsen & 2921 & 80 & 231 & 108 & 419 & 25,8 \\
\hline Brandenburg & 3050 & 48 & 214 & 157 & 419 & 37,5 \\
\hline Sachsen-Anhalt & 3678 & 5 & 92 & 122 & 219 & 55,7 \\
\hline $\begin{array}{l}\text { Mecklenburg- } \\
\text { Vorpommern }\end{array}$ & 4013 & 76 & 346 & 361 & 783 & 46,1 \\
\hline Rheinland-Pfalz & 4233 & 1605 & 642 & 59 & 2306 & 2,6 \\
\hline \begin{tabular}{|l|} 
Nordrhein- \\
Westfalen
\end{tabular} & 4426 & 35 & 156 & 205 & 396 & 51,8 \\
\hline Hessen & 5173 & 40 & 188 & 198 & 426 & 46,5 \\
\hline Saarland & 6220 & 0 & 0 & 52 & 52 & 100,0 \\
\hline $\begin{array}{l}\text { Durchschnitt }{ }^{4} \\
\text { bzw. Summe }\end{array}$ & 3468 & 3711 & 4671 & 2239 & 10621 & 21,1 \\
\hline
\end{tabular}

Quelle: Statistisches Bundesamt. 2014a und Statistisches Bundesamt. 2014b.

Anmerkungen: 1 = Die Stadtstaaten Bremen, Berlin und Hamburg werden in den amtlichen Finanzstatistiken nicht der kommunalen, sondern der staatlichen Ebene zugeordnet. 2 = Schulden je Einwohner als Summe aus Wertpapierschulden, Kassenkrediten und Krediten beim nicht-öffentlichen Bereich am 31. Dezember 2012 bezogen auf die Einwohnerzahl (auf Basis des Zensus 2011) zum 30. Juni 2012. $3=$ Schulden des öffentlichen Bereichs (ohne private Anteile) am 31. Dezember 2012 bezogen auf die Einwohnerzahl des Bundeslandes. 4 = gewichtet mit der Bevölkerung in den einzelnen Bundesländern.

Das Statistische Bundesamt (2014a) erfasst seit Kurzem die integrierten kommunalen Schulden nach dieser Systematik, und es wurden erstmals neben den Schulden der kommunalen Kernhaushalte auch die Schulden der Extrahaushalte und sonstigen öffentlichen Fonds, Einrichtungen und Unternehmen, an denen die Kommunen unmittelbar oder mittelbar beteiligt sind, einbezogen und anteilig entsprechend den Beteiligungsverhältnissen zugerechnet. Die Daten in Tabelle 1 zeigen dabei - im Aggregat - ein deutlich höheres Verschuldungsniveau der kommunalen Ebene. Die Pro-Kopf-Verschuldung steigt im Bundesdurchschnitt von 1810 
Euro (Stand 31.12.2012) um knapp das Doppelte auf 3468 Euro. Unter Einschluss der Auslagerungen und FEU ist das Bild weniger positiv. Für einige Bundesländer ergibt sich so sogar eine deutliche Änderung im Schuldenranking: So weist Ende 2012 Baden-Württemberg mit 652 die geringste Pro-Kopf-Verschuldung der Kernhaushalte auf, liegt aber unter Einschluss dieser Daten nur auf Platz vier (Tabelle 1).

\section{Abbildung 2: Schuldenstruktur der Kernhaushalte der Gemeinden (Stand 31.12.2013)}

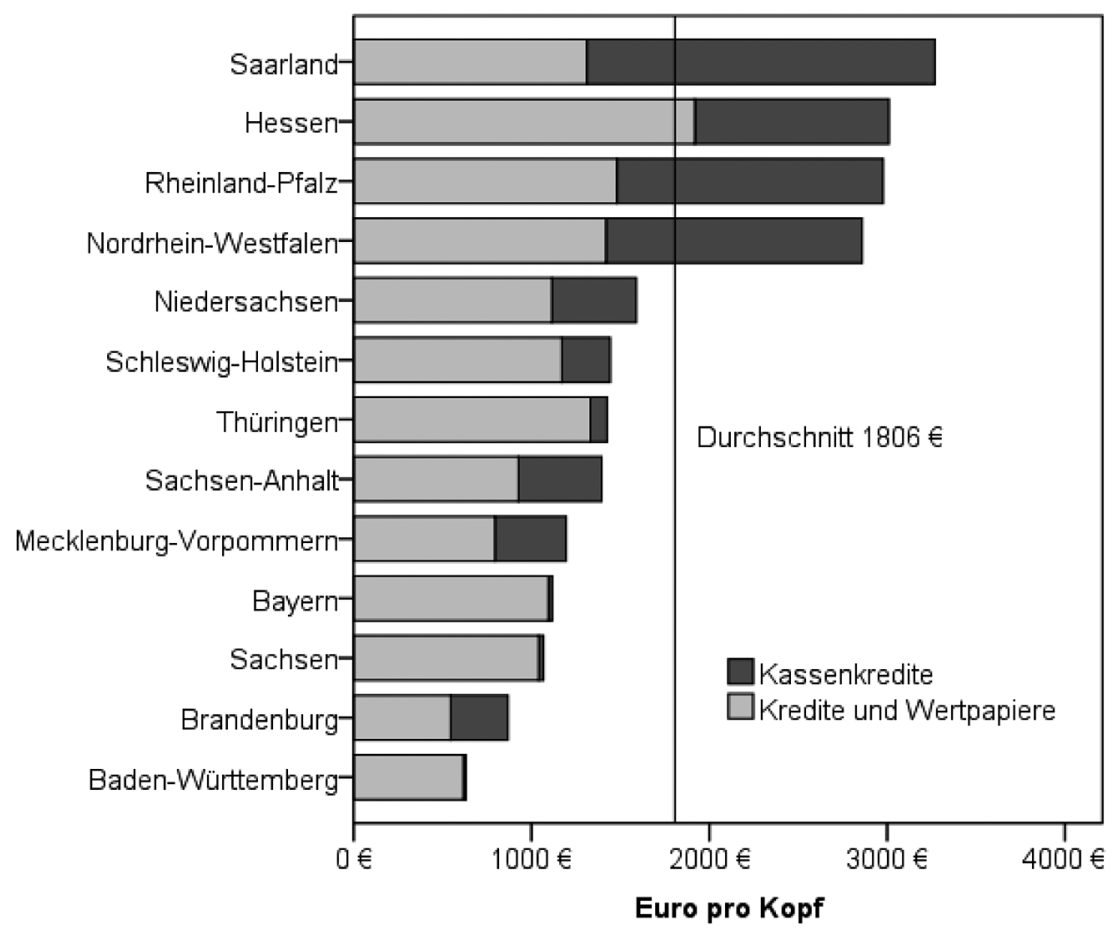

Quelle: Statistisches Bundesamt 2014b.

Anmerkung: Der Durchschnittswert ist gewichtet mit der Zahl der Einwohner. Die Stadtstaaten werden nicht betrachtet. Es werden nur die Schulden der öffentlichen Haushalte beim nicht-öffentlichen Bereich betrachtet. Schulden beim öffentlichen Sektor sowie weitere Verbindlichkeiten (z. B. Bürgschaften und kreditähnliche Rechtsgeschäfte) werden nicht betrachtet.

Eine weitere Besonderheit der Verschuldung der Kommunalebene ist die geografische Verteilung, wenn man nur die Kernhaushalte betrachtet. Auffällig ist eine deutlich unterdurchschnittliche Verschuldung in den ostdeutschen Kommunen, während alle Länder mit einer überdurchschnittlichen Kommunalverschuldung in Westdeutschland liegen. Lediglich Bayern und mit Abstand Schleswig-Holstein und Niedersachsen liegen neben Baden-Württemberg ebenfalls unter dem Bundesdurchschnitt (Abbildung 2). 
Eine weitere Perspektive ist das Verhältnis von Landes- zu kommunaler Verschuldung. Besonders sichtbar ist ein Auseinanderdriften in Schleswig-Holstein, wo die Landesverschuldung mehr als viereinhalbmal so hoch ist wie die Kommunalverschuldung. In Nordrhein-Westfalen beträgt das Verhältnis noch rund 260 Prozent, während in Sachsen das Verhältnis nur 44 Prozent beträgt, d. h. die Landesverschuldung beträgt etwa die Hälfte der Kommunalverschuldung. Dies macht deutlich, dass die Kommunalverschuldung, etwa durch unterschiedliche Aufgabenzuweisungen (Junkernheinrich u. Micosatt 2009), auch durch ein Land gesteuert werden kann. So bindet Sachsen die Kommunen im Vergleich mit Schleswig-Holstein relativ stark in die Haushaltskonsolidierung ein. Auch in Bayern ist die Landesverschuldung etwas geringer als die Gesamtheit der Verschuldung der kommunalen Ebene (rund 96 Prozent).

Ein zunehmend wichtiger Bestandteil der Schuldenanalyse ist die Schuldenstruktur. Auf der Ebene der Kommunen kann man beobachten, dass das Instrument der Kassenkredite (bzw. Liquiditätskredite) deutlich zunahm (Junkernheinrich u. Micosatt 2008; Boettcher 2013; Frankenberg u. Junkernheinrich 2014). Unter Kassenkrediten bzw. Liquiditätskrediten werden die kurzfristigen Verbindlichkeiten erfasst, welche die Berichtseinheiten zur Überbrückung vorübergehender Kassenanspannungen eingehen. Sie dienen nicht der Ausgabendeckung (keine investiven Zwecke), sondern der Aufrechterhaltung einer ordnungsgemäßen Kassenwirtschaft beziehungsweise der Liquiditätssicherung. Ende 2012 liegt der Anteil der Kassenkredite im Saarland bei über 62 Prozent, während er in RheinlandPfalz und in Nordrhein-Westfalen etwa 50 Prozent beträgt (Abbildung 2). Aus finanzwissenschaftlicher Sicht sind - längerfristig aufgenommene - Kassenkredite besonders problematisch, weil damit strukturelle Haushaltslücken (auch Personal- und Sozialausgaben) finanziert werden, denen im Gegensatz zu Investitionskrediten kein Vermögensaufbau gegenübersteht.

Damit stellt sich für die empirische Analyse die Frage nach der geeigneten abhängigen, d. h. nach der zu erklärenden, Variable. So können unterschiedliche Indikatoren verwendet werden, die dabei jeweils - aus Gründen der Vergleichbarkeit - in Euro pro Kopf gemessen werden. Dabei stehen vor allem drei Stromgrößen (d. h. jährliche Daten) und drei Bestandsgrößen (bezogen auf einen Zeitpunkt) zur Auswahl. ${ }^{4}$ Die drei wichtigsten zeitraumbezogenen Defizitgrößen sind:

(1) Der Finanzmittelsaldo, d. h. Gesamteinzahlungen abzüglich der Gesamtauszahlungen, bereinigt um besondere Finanzierungsvorgänge: Schuldenaufnahme, Tilgung, Gewährung von/Rückzahlungen aus Darlehen, Zuführungen an/ Entnahme aus Rücklagen, Fehlbeträge aus Vorjahren.

(2) das ordentliche Ergebnis im Kernhaushalt

4 Trotz der Umstellung der kommunalen Kernhaushalte auf das doppische Rechnungswesen liefert die Finanzstatistik auf absehbare Zeit keine Daten zu den gemeindlichen Haushaltsergebnissen. Vielmehr wird bei den Kommunen die Finanzrechnung erhoben. Die finanzwirtschaftliche Analyse der Kernhaushalte basiert damit „nur“ auf den Ein- und Auszahlungen in einem Jahr ohne periodengerechte Zuordnung der Zahlungen. 
(3) der Primärsaldo (bzw. das Primärergebnis): laufende Einnahmen abzgl. laufende Ausgaben einschließlich Investitionen (ohne Vermögenstransfer) und ohne Finanzierungseinnahmen/-ausgaben

Die vier Schuldenstände als Bestandsgrößen sind:

(1) die Gesamtverschuldung der Kernhaushalte

(2) die Höhe der Kassenkredite

(3) die Kreditschulden der Eigenbetriebe

(4) die konsolidierte Verschuldung der Kernhaushalte, Extrahaushalte und FEU. ${ }^{5}$

Die Analyse der kommunalen Kernhaushalte ist aufgrund des konzentrierten Verschuldungsdrucks jedoch zentral. Da die Auslagerungen in der Regel über keinen Spielraum verfügen, um sich für laufende Ausgaben zu verschulden - so droht bei privatrechtlich geführten kommunalen Unternehmen sonst die Insolvenz -, müssen etwaige Defizite aus den kommunalen Kernhaushalten finanziert werden. Die Finanzierungsprobleme der Auslagerungen verlagern sich folglich dorthin und belasten somit das Haushaltsergebnis. Der Auslagerungsgrad ist deshalb vor allem beim interkommunalen Vergleich der Aufgabenwahrnehmung von Bedeutung. Die Bewertung des Haushaltsergebnisses beschränkt sich dagegen - mit Ausnahme versteckter Risiken - zumeist auf den Kernhaushalt.

5 Trotz der Publikation konsolidierter Daten (Statistisches Bundesamt 2014a) stehen keine Zeitreihen für eine Analyse zur Verfügung, und es fehlen detaillierte Daten für Baden-Württemberg. 


\section{Abbildung 3: Finanzmittelsaldo der kommunalen Kernhaushalte in Euro je Einwohner (Durchschnitte 1991-2000 und 2001-2012)}

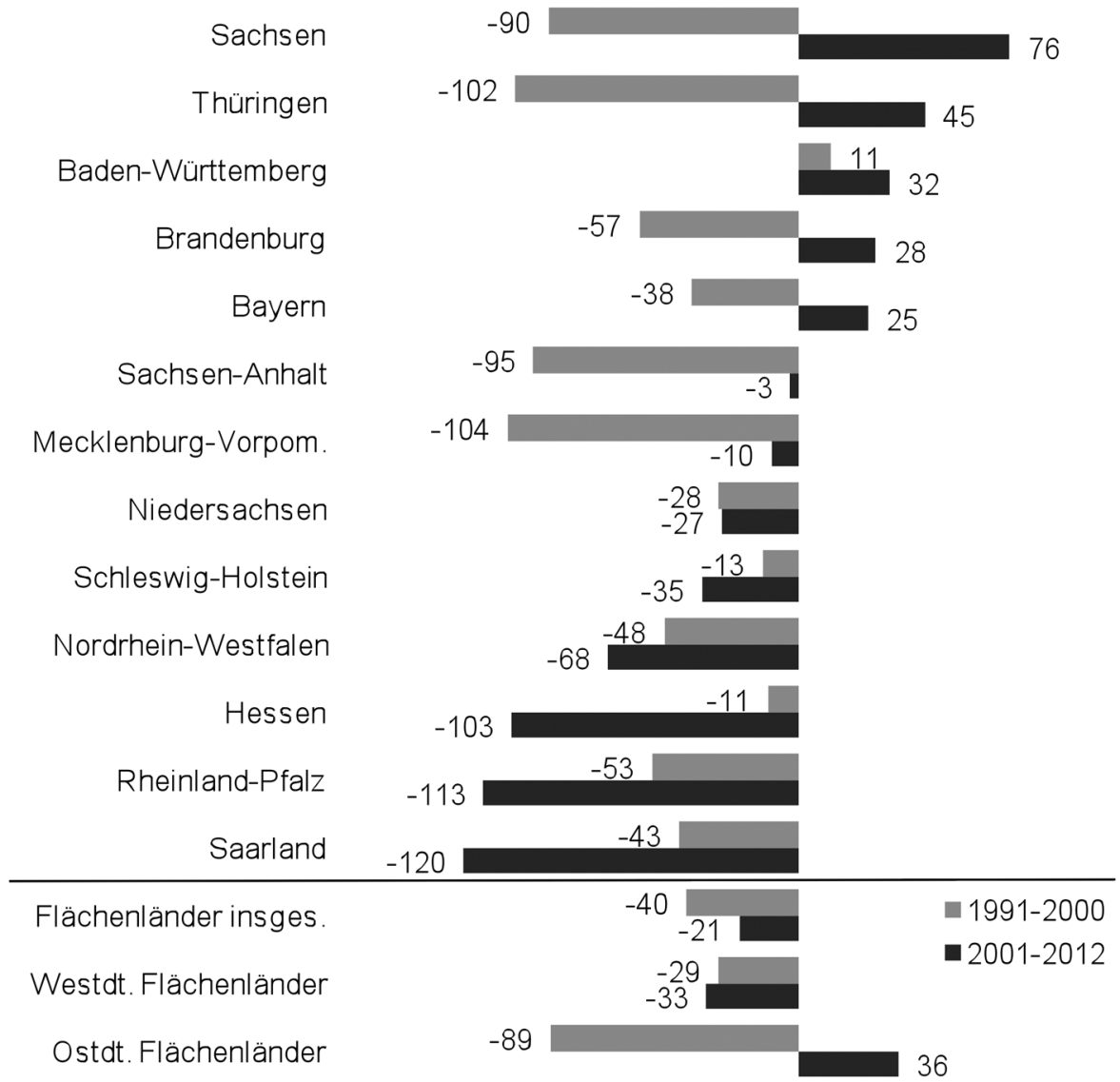

Quellen: Eigene Berechnungen nach Angaben des Statistischen Bundesamtes.

Gemessen am Finanzmittelsaldo, der zusammen mit dem Primärsaldo die abhängige Variable für die quantitative Analyse ist, stellt sich die kommunale Finanzsituation im Flächenländervergleich - wie beim Schuldenstand - ausgesprochen heterogen dar (Abbildung 3). Überdies kann zudem eine zeitliche Varianz in den letzten 20 Jahren beobachtet werden, wobei insbesondere in Ostdeutschland sich die Situation besser darstellt. Für die Periode 2001 bis 2012 weisen neben BadenWürttemberg allein die Kommunen in Bayern, Brandenburg, Niedersachsen, Sachsen-Anhalt und Thüringen - im Durchschnitt - einen Haushaltsüberschuss auf. Die saarländischen Kommunen befinden sich hingegen mit Hessen, Rheinland-Pfalz und Schleswig-Holstein sowie Nordrhein-Westfalen in einer defizitären Situation. 


\section{Hypothesen zu den Ursachen der Kommunalverschuldung}

Im Folgenden sollen die zentralen Hypothesen für die empirischen Untersuchungen formuliert und begründet werden. Eine erste Unterscheidungsmöglichkeit der Determinanten der Kommunalverschuldung bietet sich mit der Frage, ob es sich um exogene oder (politisch) endogene Faktoren der Verschuldung handelt. Exogene Faktoren sind zunächst einmal kaum durch Politiker direkt zu beeinflussen. Neben klassischen Ursachen wie Krieg, Staatszerfall und Systemwechsel beeinflussen vor allem sozioökonomische Faktoren die Staatsverschuldung. Ein starkes Wirtschaftswachstum beispielsweise bremst über mehr Steuereinnahmen und weniger Arbeitslosigkeit den Schuldenanstieg. Und umgekehrt sind makroökonomische Schocks und Krisenereignisse (Peacock u. Wiseman 1967) der Motor für Niveauverschiebungen der Staatsverschuldung nach oben. Als exogene Faktoren können auch demografische Variablen gelten. So ist etwa die Seniorenquote eine starke Triebkraft der Staatsausgaben und der Staatsverschuldung. Die traditionelle Finanzwissenschaft fokussiert vor allem auf solche exogenen, zumeist sozioökonomischen Faktoren als Verschuldungsursachen (Heinemann et al. 2009). Direkt auf die kommunale Finanzausstattung wirken die exogenen Faktoren etwa über die lokalen wirtschafts-, bevölkerungs- sowie siedlungs- und raumstrukturellen Verhältnisse. Diese zentrale „funktionalistische“ Hypothese wird dabei wie folgt formuliert:

H1: Je höher die sozioökonomische Belastung einer Kommune, desto schlechter ist der Haushaltssaldo.

Auch die kommunale Finanzausstattung (Verteilung der Auf- und Ausgaben zwischen Land und Kommunen, kommunaler Finanzausgleich) hat Auswirkungen auf die Verschuldungssituation. So zeigen Junkernheinrich und Micosatt (2009), dass es hier eine erhebliche Variation zwischen den Bundesländern gibt. Wagschal et al. können zudem zeigen, dass es eine enge Beziehung zwischen der Finanzausstattung der Kommunen und der Verschuldungsperformanz der Länder gibt (Wagschal et al. 2009). Im Prinzip sind Landes- und Kommunalverschuldung zu einem Gutteil kommunizierende Röhren.

H2: Je schlechter die Finanzausstattung einer Kommune, desto schlechter ist der Haushaltssaldo.

Sozioökonomische bzw. demografische Faktoren können andererseits auch zu endogenen Variablen werden, wenn diese polit-ökonomisch interpretiert werden. So kann die elektorale Bedeutung der Senioren ausgabensteigernd wirken, indem die Parteien - wenn an der Regierung - diese Wählergruppe besonders begünstigen. Auch Institutionen, die eigentlich von ihrer Wirkung her den exogenen Variablen zuzurechnen sind, können mitunter zu endogenen Faktoren werden, wie etwa die Kommunalaufsicht. Während im internationalen Vergleich und auf Bundesländerebene (Wagschal et al. 2009) schon länger endogene Faktoren empirisch untersucht werden, beginnt sich auch in Untersuchungen der kommunalen Ebene die Empirie zunehmend auszudifferenzieren, und insbesondere in Bezug auf Ruhrgebietskommunen wurden endogene Faktoren für Haushaltsdefizite in Fallstudien untersucht (Timm-Arnold 2011; Holtkamp 2010). 
Der wohl prominenteste endogene Faktor in der Verschuldungsliteratur ist die vermeintlich verschuldungserhöhende Wirkung von Wahlen, der auf die Theorie des politischen Konjunkturzyklus zurückgeführt werden kann (Nordhaus 1975): Regierungen gleich welcher politischer Couleur verschulden sich vor Wahlen stärker, so die von Edward Tufte (1978) erweiterte These. Jedoch zeigen neuere Studien, dass die Frage, ob politische Konjunkturzyklen tatsächlich existieren, vom Ländersample (Brender u. Drazen 2005) und von institutionellen Charakteristika der betrachteten Staaten (z. B. Demokratieniveau, politische Polarisierung, Intensität des elektoralen Wettbewerbs) abhängen.

\section{H3: Vor Kommunalwablen verschlechtert sich der Haushaltssaldo einer Kommune.}

Niskanen (1971) unterstellte als weiteren (endogenen) Einflussfaktor, dass die Bürokratie ein inhärentes Interesse an einer Maximierung ihres Einflusses besitzt, d. h. vor allem an Budgetsteigerungen. Dies kann bei mangelnder Finanzierung über höhere Steuern auch auf die Verschuldung durchschlagen. Die empirischen Ergebnisse sind jedoch nicht überzeugend, sodass der Bürokratieeinfluss auf den Verschuldungsanstieg umstritten bleibt. Auch diese Hypothese kann für die Gemeindeebene adaptiert werden.

H4a: Je höher der Anteil der Beschäftigten (bzw. je höher die Personalausgaben H4b), desto eher verschlechtert sich der Haushaltssaldo einer Kommune.

In demokratischen Systemen ist eine der zentralen Fragen im Hinblick auf Unterschiede in der Staatstätigkeit, ob Parteien einen Unterschied bei der Verschuldung machen. In Anlehnung an die Parteiendifferenztheorie von Hibbs (1977) wird man davon ausgehen, dass linke Regierungen in Krisenzeiten eine höhere Verschuldung in Kauf nehmen als rechte Regierungen, da das vorrangige Ziel linker Regierungen - gemäß ihrer Kernwählerschaft - eine niedrige Arbeitslosenquote ist. Zudem sind linke Regierungen eher der Politik des „deficit spending “ verhaftet, was als „intellektueller Motor“ für eine steigende Verschuldung angesehen werden kann (Buchanan u. Wagner 1977).

Robert Barro (1979) formuliert als zentrale These, dass die öffentlichen Finanzen durch Regierungen vorrangig so gestaltet werden, um die Steuern niedrig und möglichst wenig schwankend im Zeitablauf beizubehalten (TaxSmoothing). Folgt man dagegen der „modifizierten Steuerglättungshypothese“ (Wagschal 1996a), so werden sich bürgerliche und rechte Regierungen stärker als linke Regierungen verschulden. Analog zur Parteiendifferenzhypothese steht die Wählerbasis der Parteien wieder als treibende Kraft im Hintergrund. Bürgerliche Regierungen haben das vorrangige Ziel, die Steuerbelastung für ihre Klientel zu vermindern, was linke Regierungen nicht primär anstreben.

H5a: Je stärker Linksparteien im Gemeinderat/Kommunalparlament vertreten sind, desto schlechter ist der Haushaltssaldo einer Kommune.

H5b: Gehört der Bürgermeister einer politischen linken Partei (SPD, Linke oder Grüne) an, so fällt der Haushaltssaldo einer Kommune schlechter aus. 
H6a: Je stärker bürgerliche Parteien im Gemeinderat/Kommunalparlament vertreten sind, desto besser ist der Haushaltssaldo einer Kommune.

H6b: Gehört der Bürgermeister einer bürgerlichen Partei (CDU/CSU, FDP) an, so fällt der Haushaltssaldo einer Kommune besser aus.

Bei diesen inhaltlich gegenläufigen Hypothesen stellt sich zudem ein Kausalitätsund (methodisches) Endogenitätsproblem. So könnte es sein, dass verschuldete Kommunen eher dazu neigen, linke Bürgermeister zu wählen oder in Reaktion auf eine vergangene hohe Verschuldung einen eher rechten Bürgermeister. Dies soll mittels der Analyse über die Zeit kontrolliert werden.

Akteursbezogene Erklärungsfaktoren sind durch die erwähnte Parteiendifferenztheorie geprägt sowie durch Erklärungsansätze, in deren Zentrum die Idealtypen der Konkurrenz- und Konkordanzdemokratie stehen. In der quantitativ ausgerichteten lokalen Politikforschung konnte man bezüglich der Parteiendifferenzhypothese überwiegend keinen Zusammenhang zwischen Parteiprogrammatik und Haushaltsergebnissen auf der Kommunalebene feststellen (Wagschal 1996b; Holtkamp 2010). Dies wird u. a. mit der Tradition der primär sachbezogenen Kommunalpolitik mit geringem Professionalisierungsgrad und der Dominanz sozioökonomischer und institutioneller Variablen in der kommunalen Haushaltspolitik erklärt.

In der kommunalen Konkordanzdemokratie wird - wie in der von Lehmbruch beschriebenen nationalen Konkordanzdemokratie - das Mehrheitsprinzip durch die Einstimmigkeitsregel ersetzt (Bogumil 2001; Holtkamp 2010). Danach werden Entscheidungsprozesse zwischen den Fraktionen durch Verhandlungen und solidarische Interaktionsorientierung von der Maxime des gütlichen Einvernehmens geprägt. Diese Motivation variiert mit der Gemeindegröße. Kommunale Konkordanzdemokratie ist vor allem Ausdruck der Schwäche der Parteien bei gleichzeitiger Dominanz der Persönlichkeiten in der kommunalen Vertretungskörperschaft und des hauptamtlichen Verwaltungschefs. Sie ist also durch eine geringe personelle, prozedurale und inhaltliche Parteipolitisierung geprägt. Im Gegensatz dazu treten in der kommunalen Konkurrenzdemokratie die einzelnen Personen eher hinter einer starken Parteiorganisation und Fraktionsdisziplin zurück. Dementsprechend wird erwartet, dass bei konkurrenzdemokratischen Strukturen die Bürgermeister durch ihre Einbindung in die Parteien eine nicht so ausgeprägte Machtposition haben. Insbesondere aufgrund von Studien zur nordrhein-westfälischen Kommunalpolitik (also bei durchaus begrenzter Varianz) wird davon ausgegangen, dass konkurrenzdemokratische Konstellationen insgesamt zu höheren kommunalen Haushaltsdefiziten führen (Schulenburg 1999, S. 254; Holtkamp 2010; Timm-Arnold 2011). Der stärker ausgeprägte Parteienwettbewerb im Kommunalparlament, das Amtsverständnis der Bürgermeister mit überwiegend parteipolitischen Hintergrund und die weniger ausgeprägte exekutive Führerschaft (Machtposition der Bürgermeister) bei konkurrenzdemokratischen Konstellationen forcieren danach Haushaltsdefizite und erschweren die Haushaltskonsolidierung.

H7a: Je stärker konkordanzdemokratische Verhaltensmuster vorzufinden sind, desto besser ist der Haushaltssaldo einer Kommune. 
H7b: Je eher konkurrenzdemokratische Verhaltensmuster vorzufinden sind, desto schlechter ist der Haushaltssaldo einer Kommune.

Eng zusammenhängend mit der letzten These wird in der Literatur die Gestaltungsmacht des Bürgermeisters als Akteur immer wieder betont (Banner 1984, 1987, 2006). Die Stellung des Bürgermeisters hängt zentral von seinen Kompetenzen ab, die durch das politische System (also eine Strukturvariable) geprägt wird. Diese Kompetenzen werden in den Gemeindeordnungen vom Landesgesetzgeber erlassen. Die Gemeindeordnungen sind die Verfassungen der Gemeinden und bestimmen damit die Stellung des Bürgermeisters, seine Kompetenzen und somit auch Handlungsspielräume. Historisch gesehen gab es vier unterschiedliche Kommunalverfassungstypen (Süddeutsche Ratsverfassung, Magistratsverfassung, Norddeutsche Ratsverfassung, Rheinische Bürgermeisterverfassung), die sich durch die kommunalpolitischen Reformen der Gemeindeordnungen stark annäherten und im Wesentlichen der Süddeutschen Ratsverfassung anglichen. Dennoch gibt es weiterhin gravierende Unterschiede in den Kommunalverfassungen. Bogumil und Holtkamp (2013) haben die Gemeindeordnungen der Flächenländer im Zeitraum 2003 bis 2012 ausgewertet, dabei die Kompetenzen des Bürgermeisters sowie des Gemeinderats empirisch erfasst und zu einem Kommunalverfassungsindex zusammengefügt (Bogumil u. Holtkamp 2013, S. 30). Die Hypothese in den Untersuchungen lautet daher:

H8: Je stärker die Machtstellung des Bürgermeisters, desto besser ist der Haushaltssaldo einer Kommune.

Die folgenden empirischen Analysen fokussieren daher auf folgende zentrale unabhängige Variablenkategorien: (1) sozioökonomische, (2) institutionelle, (3) parteipolitische sowie (4) sich aus der Handlungsorientierung und Interaktion der Akteure ergebende Faktoren.

\section{Ergebnisse der quantitativen Analyse}

Bei einer Umfrage zur Haushaltssituation in den Kommunen (Wagschal u. Grasl 2014) unter allen Bürgermeistern Deutschlands in Städten ab 10.000 Einwohnern (Stand 2013) wurde gefragt, welche externen Faktoren besonders auf die Haushaltspolitik einwirken. Mit über 54 Prozent war die Rücklaufquote dieser Umfrage beachtlich. Aus Sicht der Bürgermeister sind die externen Faktoren vor allem auf der Einnahmenseite zu verorten (Abbildung 4). So wird der kommunale Finanzausgleich als zu gering erachtet, ebenso wie die kommunale Finanzausstattung sowie die Schlüsselzuweisungen. Daran anschließend folgen die zu zahlreichen Aufgaben sowie hohe Sozialausgaben. Die demografische Entwicklung wird von den neun abgefragten Items als am wenigsten wichtig gesehen. Ebenso kommt der wirtschaftlichen Entwicklung aus Sicht der Bürgermeister nur eine geringe Bedeutung zu. Dies widerspricht zumindest den empirischen Befunden im internationalen Vergleich (Wagschal 1996a) oder auch im Bundesländervergleich (Wagschal et al. 2009), die in Regressionsanalysen immer mit die höchste Erklärungskraft aufwiesen. 


\section{Abbildung 4: Der Einfluss externer Faktoren auf die Haushaltspolitik}

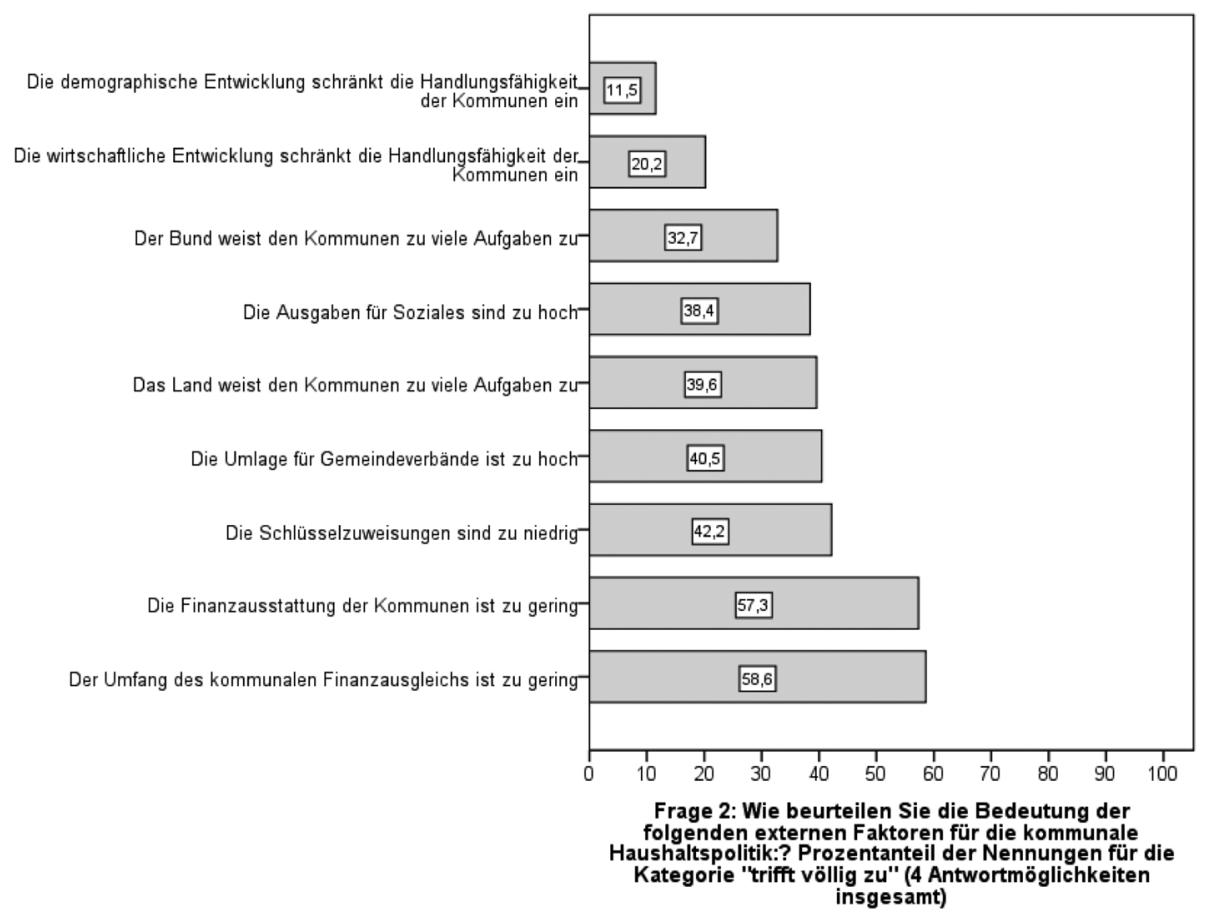

Quelle: Bürgermeisterbefragung 2013 (Wagschal u. Grasl 2014). 810 Bürgermeister in Kommunen über 10.000 Einwohner beantworteten diese Frage.

Belastbarer als die Befragung der Entscheidungsträger und die Auswertung bivariater Zusammenhänge ist jedoch die Analyse der Determinanten in multivariaten Regressionsanalysen. In Tabelle 2 werden nun unterschiedliche exogene und endogene Einflussfaktoren gleichzeitig in einer Querschnittsregression zum Zeitpunkt 2010 getestet, um die Bedeutung der wichtigsten Verschuldungsfaktoren zu identifizieren. Dabei werden zwei abhängige Variablen betrachtet: Die kommunale Verschuldung im Kernhaushalt (Gleichung 1 und 2) sowie der Primärsaldo des Kernhaushalts, als Differenz der laufenden Ausgaben und Einnahmen (Gleichung 3 und 4).

In Tabelle 2 werden jeweils die Modelle für den Querschnitt zum Zeitpunkt 2010 geschätzt. Insofern stellen die Befunde eine Momentaufnahme dar und lassen nur bedingt Rückschlüsse auf längere Zeiträume zu. Aus Gründen der Datenverfügbarkeit werden nur Städte und Gemeinden mit mehr als 10.000 Einwohner betrachtet, was in der Summe immerhin über 60 Millionen Menschen in Deutschland (= 73 Prozent der Bevölkerung) einschließt. ${ }^{6}$

6 Insgesamt weisen zum Zeitpunkt 20101567 Gemeinden und Städte mehr als 10.000 Einwohner auf. Durch fehlende Werte werden in der Regression 456 Kommunen weniger betrachtet. Diese 1111 Städte und Gemeinden repräsentieren aber immerhin noch 45 Millionen Einwohner (= 55 Prozent der Gesamtbevölkerung). 
Tabelle 2: Regressionsmodelle zur Erklärung der Gesamtverschuldung im Kernhaushalt (2010) und des Primärergebnisses der Kommunen (2010)

\begin{tabular}{|c|c|c|c|c|}
\hline & $\begin{array}{c}(1) \\
\text { Schulden im } \\
\text { Kernhaushalt }\end{array}$ & $\begin{array}{c}(2) \\
\text { Schulden im } \\
\text { Kernhaushalt }\end{array}$ & $\begin{array}{c}(3) \\
\text { Primärsaldo }\end{array}$ & $\begin{array}{c}(4) \\
\text { Primärsaldo }\end{array}$ \\
\hline $\begin{array}{l}\text { Anteil der Empfänger } \\
\text { von Grundsicherung }\end{array}$ & $\begin{array}{c}18,2 * * * \\
(2,92)\end{array}$ & $\begin{array}{c}46,7 * * * \\
(5,38)\end{array}$ & $\begin{array}{c}-1,26 \\
(-0,43)\end{array}$ & $\begin{array}{l}-3,02 \\
(0,99)\end{array}$ \\
\hline Bedeutung als Arbeitsort & $\begin{array}{c}44,4 * * * \\
(0,56)\end{array}$ & $\begin{array}{l}46,7 * * * \\
(-4,28)\end{array}$ & $\begin{array}{c}-17,8 \\
(-0,65)\end{array}$ & $\begin{array}{l}-11,68 \\
(-0,42)\end{array}$ \\
\hline Erwerbstätigenquote & $\begin{array}{l}-18,6^{* *} \\
(-2,74)\end{array}$ & $\begin{array}{l}-10,2 \\
(1,44)\end{array}$ & $\begin{array}{r}-3,38 \\
(-1,42)\end{array}$ & $\begin{array}{l}-4,17^{*} \\
(-1,70)\end{array}$ \\
\hline $\begin{array}{l}\text { Verschuldung im } \\
\text { Kernhaushalt }\end{array}$ & & & $\begin{array}{l}-0,02 * \\
(-2,10)\end{array}$ & $\begin{array}{l}-0,02 * \\
(-1,96)\end{array}$ \\
\hline $\begin{array}{l}\text { Gewerbesteuer- } \\
\text { aufkommen }\end{array}$ & $\begin{array}{l}-0,35^{* *} \\
(-4,58)\end{array}$ & $\begin{array}{l}-0,32 * * * \\
(-4,32)\end{array}$ & $\begin{array}{c}0,03 \\
(0,98)\end{array}$ & $\begin{array}{c}0,04 \\
(1,53)\end{array}$ \\
\hline $\begin{array}{l}\text { West- oder Ostdeutsch- } \\
\text { land }(0 / 1)\end{array}$ & & $\begin{array}{c}-544,8 * \cdots \\
(4,33)\end{array}$ & & $\begin{array}{c}104,2 * * \\
(2,36)\end{array}$ \\
\hline $\begin{array}{l}\text { Personalausgaben } \\
\text { Kernhaushalt }\end{array}$ & $\begin{array}{c}0,54 * * * \\
(3,50)\end{array}$ & $\begin{array}{c}0,50 * * * \\
(3,24)\end{array}$ & $\begin{array}{l}-0,44 * * * \\
(-8,18)\end{array}$ & $\begin{array}{l}-0,45 * * \\
(-8,30)\end{array}$ \\
\hline $\begin{array}{l}\text { Kommunalverfassungs- } \\
\text { index }\end{array}$ & $\begin{array}{c}-51,1^{* * *} \\
(4,83)\end{array}$ & $\begin{array}{l}-42,7^{* * *} \\
(-3,94)\end{array}$ & $\begin{array}{c}6,02 \\
(1,64)\end{array}$ & $\begin{array}{c}4,84 \\
(1,38)\end{array}$ \\
\hline $\begin{array}{l}\text { Zersplitterung des Ge- } \\
\text { meindeparlaments (Rae- } \\
\text { Index) }\end{array}$ & $\begin{array}{l}566,9 \\
(1,51)\end{array}$ & $\begin{array}{l}675,4 \\
(1,56)\end{array}$ & $\begin{array}{c}-41,8 \\
(-0,32)\end{array}$ & $\begin{array}{l}-111,1 \\
(-0,74)\end{array}$ \\
\hline SPD-Bürgermeister $(0 / 1)$ & & $\begin{array}{c}88,2 \\
(1,46)\end{array}$ & & $\begin{array}{c}1,34 \\
(0,06)\end{array}$ \\
\hline $\begin{array}{l}\text { Größe des Gemeinderates/ } \\
\text { Stadtparlaments }\end{array}$ & $\begin{array}{c}29,0 * * * \\
(9,06)\end{array}$ & $\begin{array}{c}26,1 * * * \\
(8,09)\end{array}$ & $\begin{array}{l}2.17^{*} \\
(1,90)\end{array}$ & $\begin{array}{c}2,70 * * \\
(2,33)\end{array}$ \\
\hline CDU-Bürgermeister (0/1) & $\begin{array}{c}7,4 \\
(0,14)\end{array}$ & & $\begin{array}{l}11,9 \\
(0,66)\end{array}$ & \\
\hline $\begin{array}{l}\text { CDU-Sitzanteil im } \\
\text { Stadtparlament }\end{array}$ & & $\begin{array}{r}-2,00 \\
(-0,65)\end{array}$ & & $\begin{array}{c}-0,73 \\
(-0,68)\end{array}$ \\
\hline $\begin{array}{l}\text { Sitzanteil linker Parteien } \\
\text { im Stadtparlament }\end{array}$ & $\begin{array}{c}3,18 \\
(1,34)\end{array}$ & & $\begin{array}{l}3,57^{*} \\
(1,90)\end{array}$ & \\
\hline Konstante & $\begin{array}{c}1025,4 * * \\
(2,19)\end{array}$ & $\begin{array}{c}1061,2 * \\
(1,93)\end{array}$ & $\begin{array}{l}164,2 \\
(0,97) \\
\end{array}$ & $\begin{array}{l}194,8 \\
(1,02) \\
\end{array}$ \\
\hline Fallzahl & 1111 & 1111 & 1111 & 1111 \\
\hline F-Statistik & $49,04 * * *$ & $47,60 * * *$ & $10,56 * *$ & $9,83 * *$ \\
\hline Höchster VIF-Wert & 1,98 & 3,00 & 2,78 & 3,08 \\
\hline Korrigiertes $\mathrm{R}^{2}$ & 0,30 & 0,32 & 0,09 & 0,09 \\
\hline
\end{tabular}

Quelle: Eigene Berechnungen

Anmerkungen: Angegeben sind die OLS-Regressionskoeffizienten sowie in Klammern die t-Werte. Höchster VIF-Wert ist der höchste Varianzinflationsfaktor unter allen unabhängigen Variablen. ${ }^{* * *}:$ signifikant auf dem 1-Prozent-Niveau, ${ }^{* *}$ : signifikant auf dem 5-Prozent-Niveau, ${ }^{*}$ : signifikant auf dem 10-Prozent-Niveau. Abhängige Variablen: Verschuldung im Kernhaushalt (Gleichung 1 und 2); Primärergebnis (Gleichung 3 und 4). 
Bei der Erklärung des kommunalen Verschuldungsstandes sind die exogenen Faktoren besonders erklärungskräftig. Es zeigt sich, dass der Anteil der Empfänger von Grundsicherungsleistungen (SGB-II-Quote) einen deutlichen Einfluss auf die Höhe der kommunalen Verschuldungsniveaus besitzt. Daneben ist auch die Bedeutung als Arbeitsort (erfasst über die Pendlerströme) relevant, der verschuldungserhöhend wirkt. Je mehr Erwerbstätige eine Kommune hat - eine Näherungsgröße für die Wirtschaftsstärke -, desto niedriger ist der akkumulierte Schuldenstand (signifikant aber nur in Gleichung 1).

Daneben zeigt sich auch, dass die Ausgabenstruktur der Haushalte, insbesondere die Personalausgaben, einen wichtigen Erklärungsbeitrag für die Höhe der kommunalen Schuldenstände und des Haushaltsdefizits (Primärsaldo) liefern. Je höher die Personalausgaben, desto höher die Verschuldung. Von den soziökonomischen Faktoren können aufgrund der Multikollinearität nicht alle im Datensatz erfassten Faktoren geprüft werden, da diese insbesondere mit dem Anteil der Empfänger der Grundsicherung hoch korrelieren. Hierzu zählt etwa der Anteil der Arbeitslosen in einer Kommune oder auch die Höhe der Kaufkraft. Generell kann man jedoch feststellen, dass es gerade soziökonomische Problemlagen sind, die die Verschuldung besonders beeinflussen, was auch über die Höhe der BetaGewichte bestätigt wird.

Auf der Einnahmeseite zeigt sich, dass die Höhe der Gewerbesteuereinnahmen einen deutlichen Einfluss auf die Höhe des Verschuldungstandes hat und sich hierauf dämpfend auswirkt. Die eigene finanzielle Leistungsfähigkeit einer Kommune ist trotz der Umverteilung im kommunalen Finanzausgleich ein wichtiges Element zur Sicherstellung der finanziellen Unabhängigkeit von Kommunen.

Bei dem Primärsaldo zeigt sich - schwach signifikant - die Bedeutung der Gesamtverschuldung. Diese Pfadabhängigkeit bedeutet, dass Kommunen, die bereits eine hohe Verschuldung in den vergangenen Jahren akkumulierten, jedes Jahr zusätzliche höhere Kassenkreditverschuldung aufnehmen müssen. Dies heißt, dass sich die Verschuldung in den hochverschuldeten Kommunen weiter verfestigt.

Die endogenen Faktoren, also die Parteizugehörigkeit der Bürgermeister oder etwa auch der Sitzanteil einzelner Parteien wie etwa der SPD, der CDU oder der Wählergruppen, sind im Großen und Ganzen bei der Querschnittsanalyse insignifikant. Lediglich die aggregierte Stärke der Linksparteien (SPD, Grüne, Linke) wirkt eher positiv auf den Primärsaldo. Parteipolitische Faktoren spielen demnach, wenn man für die anderen theoretisch relevanten Faktoren kontrolliert, keine Rolle. Dagegen ist besonders die Kommunalverfassung relevant. Je stärker die institutionelle Stellung von Bürgermeistern ist, desto niedriger liegt die Kommunalverschuldung. Gerade mit der Süddeutschen Ratsverfassung zeigt sich dieser Effekt besonders. Daneben weist die Größe des Gemeinderates bzw. des Stadtparlaments durchgehend einen verschuldungserhöhenden Effekt auf. Die Größe des Kommunalparlaments hängt zwar mit der Gemeindegröße zusammen (und berücksichtigt daher auch den thematisierten Größeneffekt der Verschuldung), aber inhaltlich kann der Kausalmechanismus nachvollzogen werden. Je mehr Gemeinderäte, desto höher die Verschuldung, da sich die Legitimation oftmals über erreichte Ausgaben speist. Für das Haushaltsergebnis ist dieser Faktor jedoch insignifikant. 
Generell ist die Erklärungskraft der Modelle für das jährliche Primärergebnis schwach. Ein Großteil der Varianz bleibt unaufgeklärt. Im Hinblick auf die relative Wichtigkeit der einzelnen Erklärungsfaktoren (beurteilt über die Beta-Werte) zeigt sich, dass für den Verschuldungsstand der Anteil der Empfänger von Grundsicherungsleistungen, die Größe des Kommunalparlaments, die West-Ost-Lage, die Höhe der Gewerbesteuer und die Ausgestaltung der Kommunalverfassung die relevantesten Faktoren sind. Für das Haushaltssaldo (Primärsaldo) sind die folgenden Faktoren - auf Basis der Beta-Werte - am relevantesten: die Höhe der Personalausgaben, die West-Ost-Lage, die Größe des Stadtparlaments sowie die bestehende Verschuldung im Kernhaushalt.

Erklärungen für die geringe Erklärungskraft der exogenen und endogenen Faktoren könnten die verschiedenen Ausgleichsmechanismen auf kommunaler Ebene sein, die insgesamt nivellierend wirken oder auch, dass verschiedene Faktoren, die sich in den Interviews und Befragungen zu dieser Studie als relevant erwiesen (vor allem die Kommunalaufsicht), noch nicht empirisch quantifiziert wurden.

In Tabelle 3 wird die bisherige einjährige Querschnittsanalyse um ein Regressionsmodell mit Panel-Daten erweitert. Dabei werden zwei unterschiedliche abhängige Variablen betrachtet: (1) Der Finanzmittelsaldo, d. h. Gesamteinzahlungen abzüglich der Gesamtauszahlungen, also der entscheidende Indikator, ob eine Kommune ein Defizit oder einen Überschuss erwirtschafteten konnte (Frankenberg u. Junkernheinrich 2014) und (2) der Primärsaldo, d. h. Einnahmen abzüglich Ausgaben der laufenden Verwaltung einer Kommune. Der Primärsaldo beschränkt sich stärker auf das laufende Geschäft. Beispielsweise werden hier einzelne Vermögenstransfers nicht erfasst.

Der Paneldatensatz umfasst aus Gründen der Datenverfügbarkeit die Jahre 2003 bis 2010. In der Betrachtung stehen Gemeinden mit einer Mindesteinwohnerzahl von 20.000. Diese Grenze wird gezogen, da die Verfügbarkeit der Daten darunter abnimmt und die Heterogenität mit schrumpfender Bevölkerungszahl stark zunimmt. Als erklärende Faktoren werden zunächst sozioökonomische Größen der Kommunen betrachtet. Hierunter fallen die Bevölkerungszahl, die Erwerbstätigenquote (gemessen als inverser Sozialbelastungsparameter ${ }^{7}$ sowie als Indikator für die Wirtschaftskraft die Kaufkraft). Außerdem wird der Zentralitätsparameter Bedeutung als Arbeitsort in die Regressionsanalyse aufgenommen. Dieser ergibt sich aus der Division aus den sozialversicherungspflichtig Beschäftigten am Arbeitsort und den sozialversicherungspflichtig Beschäftigten am Wohnort. Somit hat eine Kommune mehr Einpendler als Auspendler, wenn dieser Wert größer als 1 ist. Da die Werte dieser Größe im Minimum gegen 0 gehen und im Maximum bei 5 liegen, sind hier besonders hohe Koeffizienten zu erwarten. Als Näherungsgröße für die Wirtschaftsstruktur einer Kommune fließt der Anteil der Erwerbstätigen im Dienstleistungssektor in die Regressionsanalyse ein. Zusätzlich könnte auch die Altersstruktur einer Kommune Auswirkungen auf deren Defizite

7 Die eigentlichen Belastungsparameter Arbeitslosenquote und SGB II-Quote werden aufgrund der geringen Verfügbarkeit über die acht Jahre nicht mit einbezogen. Schätzungen mit der Arbeitslosenquote wurden jedoch auch durchgeführt. Die Ergebnisse weichen nicht stark ab und können auf Anfrage nachgeliefert werden. 
haben. Somit wird auch der Altenquotient, welcher durch den Anteil der über 65-Jährigen Menschen im Vergleich zu dem Anteil der 20- bis 64-Jährigen definiert ist, berücksichtigt.

Als weiterer Bestandteil fließen verschiedene politökonomische Variablen mit in die Schätzgleichung ein. Hierbei handelt es sich um die schon zuvor in der Querschnittsanalyse für das Jahr 2010 getesteten Parameter: Die Größe des Gemeinderates, die Zersplitterung des Gemeindeparlaments (Rae-Index), der CDUsowie SPD-Sitzanteil im Stadtparlament sowie jeweils zwei Dummy-Variablen für die Parteizugehörigkeit des Bürgermeisters (CDU und SPD).

Da sich im Datensatz sowohl kreisangehörige Gemeinden als auch kreisfreie Städte befinden, wird der kreisfreie Stadt-Dummy als notwendige Kontrollvariable eingeführt. Damit wird der Tatsache Rechnung getragen, dass heterogene Aufgabenbelastungen zugrunde liegen. So sind beispielsweise die kreisfreien Städte und Landkreise Hauptträger der Finanzierung sozialer Leistungen (Junkernheinrich 2012). Dementsprechend findet dieser Dummy in allen Schätzungen Anwendung.

Die Verwendung von fixen Effekten für die ökonometrische Auswertung von Panel-Datensätzen ist dann angebracht, wenn individuelle, zeitlich konstante Heterogenität korreliert ist mit einer oder mehreren unabhängigen Variablen. Ansonsten droht eine Verfälschung der Ergebnisse, da die Schätzergebnisse aufgrund der Vernachlässigung solcher Unterschiede und weiterer Faktoren, die mit der abhängigen und einer oder mehreren unabhängigen Variablen zusammenhängen, unter- oder überschätzt werden können (Omitted Variable Bias [Stock und Watson 2003]). Starke Heterogenität zwischen den Flächenländern besteht beispielsweise durch den Kommunalisierungsgrad eines Landes, also dem Anteil öffentlicher Aufgaben bzw. Ausgaben, der durch die kommunale Ebene ausgeführt wird (Junkernheinrich 2009, S. 204) oder auch durch den haushaltsrechtlichen Ordnungsrahmen eines Landes (Junkernheinrich et al. 2014). Um generell für solche flächenlandspezifischen Unterschiede, die zum Teil nicht beobachtbar oder messbar sind, zu kontrollieren, werden in allen Spezifikationen fixe Flächenlandeffekte verwendet. $^{8}$

Zusätzlich dazu werden fixe Zeiteffekte verwendet (Greene 2008, S. 193-199). Hierdurch soll für kommuneneinheitliche Unterschiede über die Zeit kontrolliert werden. Hiermit werden beispielsweise bundesweite Schocks oder jahresspezifische Veränderungen aufgefangen. Da Heteroskedastizität und auch Autokorrelation der Fehlerterme für das vorliegende Panel nicht ausgeschlossen werden können, werden robuste und geclusterte Standardfehler auf der Kommunenebene in allen Spezifikationen verwendet (Petersen 2008, S. 475-476). Somit wird für eine potenzielle Korrelation der Fehlerterme innerhalb einer Kommune über die Zeit kontrolliert.

Tabelle 3 zeigt in den Spalten 1 und 2 Schätzungen mit dem Finanzmittelsaldo als abhängige Variable und in den Spalten 3 und 4 mit dem Primärsaldo als abhängige Variable. Jeweils in Spalte 1 und 3 werden der SPD-Sitzanteil im Gemein-

8 Der durchgeführte „Hausman Test“ bestätigte, dass fixe Effekte einem Random Effects-Ansatz vorzuziehen sind (Wooldridge 2013, S. 496). 
derat sowie der SPD-Bürgermeister ausgelassen, da diese beiden sich stark invers zum jeweiligen CDU-Pendant verhalten und demnach (aufgrund von Multikollinearität) nicht in einer Schätzung verwendet werden sollten. In den Schätzungen 2 und 4 werden dementsprechend die CDU-Variablen ausgelassen.

Die Variablen Erwerbstätigenquote und Erwerbstätigenanteil im Dienstleistungssektor sind in allen Spezifikationen insignifikant. Ansonsten zeigen sich die berücksichtigten sozioökonomischen Größen durchaus als einflussreich. So wirken sich die Variablen Kaufkraft und Bedeutung als Arbeitsort stark positiv signifikant auf Finanzmittelsaldo und Primärsaldo aus. Eine Erhöhung der Kaufkraft um 1000 Euro führt hiernach zu einem Anstieg des Finanzmittelsaldos zwischen drei und vier Euro, während eine um 0,1 höhere Bedeutung als Arbeitsort mit einem um ca. sechs Euro je Einwohner höherem Finanzmittelsaldo einhergeht (Spalten 1 und 2). Demgegenüber wirkt der Altenquotient defiziterhöhend. Dessen Koeffizient ist durchgehend signifikant auf dem 1-Prozent-Niveau. Je älter also eine Kommune, desto kleiner der Finanzmittel-/Primärsaldo. Die Bevölkerungszahl hat einen schwach positiven, signifikanten Einfluss auf den Finanzmittelsaldo und ist in den Schätzungen zum Primärsaldo insignifkant. 
Tabelle 3: Panelregressionsmodell zur Erklärung kommunaler Haushaltsdefizite

\begin{tabular}{|c|c|c|c|c|}
\hline \multirow[b]{2}{*}{ Variablen } & (1) & (2) & (3) & (4) \\
\hline & FM-Saldo & FM-Saldo & Primärsaldo & Primärsaldo \\
\hline \multirow{2}{*}{ Bevölkerung } & $0,116^{*}$ & $0,120 *$ & 0,052 & 0,051 \\
\hline & $(1,718)$ & $(1,780)$ & $(0,889)$ & $(0,872)$ \\
\hline \multirow{2}{*}{$\begin{array}{l}\text { Erwerbstätigen- } \\
\text { quote }\end{array}$} & 1,618 & 1,869 & $-0,235$ & $-0,075$ \\
\hline & $(1,387)$ & $(1,592)$ & $(-0,218)$ & $(-0,070)$ \\
\hline Kaufkraft & $\begin{array}{c}0,004 * * * \\
(4,266)\end{array}$ & $\begin{array}{c}0,003 * * * \\
(3,924)\end{array}$ & $\begin{array}{c}0,003 * * * \\
(4,121)\end{array}$ & $\begin{array}{c}0,003 * * * \\
(4,019)\end{array}$ \\
\hline Bedeutung als & $59,967 * * *$ & $59,856 * * *$ & $43,845 * * *$ & $44,560 * * *$ \\
\hline Arbeitsort & $(4,657)$ & $(4,560)$ & $(3,805)$ & $(3,804)$ \\
\hline \multirow{2}{*}{$\begin{array}{l}\text { Erwerbstätigen- } \\
\text { anteil im Dienst- } \\
\text { leistungssektor }\end{array}$} & 0,032 & $-0,003$ & $-0,011$ & $-0,031$ \\
\hline & $(0,079)$ & $(-0,008)$ & $(-0,031)$ & $(-0,085)$ \\
\hline \multirow{2}{*}{ Altenquotient } & $-3,728 * * *$ & $-3,764 * * *$ & $-3,531 * * *$ & $-3,615 * * *$ \\
\hline & $(-4,102)$ & $(-4,171)$ & $(-4,088)$ & $(-4,152)$ \\
\hline Größe des & $-1,870 * * *$ & $-1,723 * *$ & $-1,301 * *$ & $-1,179 *$ \\
\hline $\begin{array}{l}\text { Gemeinderates/ } \\
\text { Stadtparlaments }\end{array}$ & $(-2,855)$ & $(-2,641)$ & $(-2,144)$ & $(-1,947)$ \\
\hline Zersplitterung des & 76,045 & 31,042 & 56,577 & $-12,897$ \\
\hline $\begin{array}{l}\text { Gemeindeparla- } \\
\text { ments (Rae-Index) }\end{array}$ & $(0,921)$ & $(0,462)$ & $(0,758)$ & $(-0,191)$ \\
\hline $\begin{array}{l}\text { Kreisfreie Stadt } \\
(0 / 1)\end{array}$ & $\begin{array}{c}-54,299 * * * \\
(-2,869)\end{array}$ & $\begin{array}{c}-51,950 * * * \\
(-2,735)\end{array}$ & $\begin{array}{c}-50,008 * * \\
(-3,068)\end{array}$ & $\begin{array}{c}-48,337 * * * \\
(-2,959)\end{array}$ \\
\hline CDU-Sitzanteil im & 0,081 & & 0,427 & \\
\hline Stadtparlament & $(0,143)$ & & $(0,825)$ & \\
\hline $\begin{array}{l}\text { CDU-Bürger- } \\
\text { meister }(0 / 1)\end{array}$ & $\begin{array}{c}28,134 * * * \\
(3,189)\end{array}$ & & $\begin{array}{c}19,933 * * \\
(2,545)\end{array}$ & \\
\hline $\begin{array}{l}\text { SPD-Bürger- } \\
\text { meister }(0 / 1)\end{array}$ & & $\begin{array}{c}-28,570 * * * \\
(-2,997)\end{array}$ & & $\begin{array}{l}-20,689 * * \\
(-2,429)\end{array}$ \\
\hline SPD-Sitzanteil im & & $-0,379$ & & $-0,224$ \\
\hline Stadtparlament & & $(-0,701)$ & & $(-0,441)$ \\
\hline Konstante & $\begin{array}{c}-260,537 * * * \\
(-2,624)\end{array}$ & $\begin{array}{c}-196,331 * * \\
(-2,192)\end{array}$ & $\begin{array}{c}-163,409^{*} \\
(-1,882)\end{array}$ & $\begin{array}{l}-83,546 \\
(-1,011)\end{array}$ \\
\hline Beobachtungen & 5.038 & 5.038 & 5.038 & 5.038 \\
\hline Korrigiertes $\mathrm{R}^{2}$ & 0,146 & 0,146 & 0,160 & 0,160 \\
\hline
\end{tabular}

Quelle: Eigene Berechnungen.

Anmerkungen: OLS-Schätzungen mit fixen Zeit- und Flächenlandeffekten; in den Klammern sind tStatistiken gegeben; Standardfehler sind robust und nach Kommunen geclustert; die Sternchen kennzeichnen Signifikanz auf dem $\left.10\left({ }^{*}\right), 5^{(* *}\right)$ und $1\left(^{* * *}\right)$ Prozent-Niveau. 
Bei den politökonomischen Größen zeigen sich die Variablen Zersplitterung des Gemeindeparlaments sowie Sitzanteile CDU/SPD als insignifikant. Zu erwähnen ist jedoch, dass sich das nach Hypothese 5a erwartet negative Vorzeichen für SPD-Sitzanteile (von der Tendenz her defiziterhöhend) und nach Hypothese 6a das erwartet positive Vorzeichen für CDU-Sitzanteile (also von der Tendenz her defizitsenkend) einstellt. Jedoch sind nur Aussagen über die Tendenz möglich, und auch die Hypothesen können nicht nachhaltig bestätigt werden, da alle Koeffizienten insignifikant sind.

Demgegenüber können die Hypothesen $5 \mathrm{~b}$ und $6 \mathrm{~b}$ bestätigt werden. In Schätzung 1 hat die Dummy-Variable für den CDU-Bürgermeister einen signifikanten Einfluss auf dem 1-Prozent-Niveau. Das Vorzeichen des Koeffizienten ist positiv. Somit ist in Kommunen, deren Bürgermeister der CDU angehört, der Finanzmittelsaldo im Durchschnitt um ca. 28,1 Euro je Einwohner höher als in Kommunen mit Bürgermeister anderer Parteizugehörigkeit. Gleichzeitig weist die Dummy-Variable für SPD-Bürgermeister einen starken, signifikant negativen Einfluss auf. Der Koeffizient von ca. 28,6 zeigt den stark inversen Charakter der beiden Variablen auf. Ähnliche Effekte lassen sich in den Spalten 3 und 4 beobachten, wobei hier die Koeffizienten etwas kleiner sind und das Signifikanzniveau auf 5 Prozent abfällt.

Die Größe des Gemeindeparlaments übt durchgehend einen negativen Einfluss auf Finanzmittel- und Primärsaldo aus. Der Einfluss auf den Finanzmittelsaldo zeigt sich als stark signifikant, während das Signifikanzniveau in den Schätzungen zum Primärsaldo nur als schwach einzustufen ist. Nach Schätzungen 1 und 2 sinkt also der Primärsaldo um ca. 1,8 Euro je Einwohner, wenn sich das Stadtparlament um ein Ratsmitglied erhöht. Da ein Zusammenhang zwischen der Bevölkerungszahl einer Kommune und deren Gemeinderatsgröße besteht, wird bewusst die Bevölkerungszahl in denselben Schätzungen eingeschlossen, um auszuschließen, dass die Variable Gemeindegröße lediglich einen Größeneffekt beschreibt. Die Korrelation dieser beiden Größen liegt bei 0,65 , jedoch ist auch nach einschlägigen Tests $^{9}$ keine erhöhte Kollinearität zu erwarten.

Die politischen Variablen weisen in den Schätzungen teilweise recht hohe Signifikanzniveaus auf. In den Spezifikationen wurde anhand der ausgewählten Variablen sowie der Verwendung von fixen Flächenland- und Zeiteffekten versucht, zu verhindern, dass Variablen fehlen, die Determinanten der abhängigen Variablen und zugleich korreliert mit den entsprechenden politischen Variablen sind (oben beschriebenes Problem des Omitted Variable Bias). So ist beispielsweise denkbar, dass besonders in Kommunen mit einer hohen sozialen Belastung vermehrt SPDBürgermeister gewählt wurden und nicht die Parteizugehörigkeit des Bürgermeisters, sondern die Sozialbelastung den Ausschlag für die zusätzliche Verschuldung gab. Aus diesem Grund wurden die Schätzungen auch unter Einbeziehung der SGB II-Quote, die für wenige Jahre vorliegt und deshalb in den dargestellten Schätzungen nicht berücksichtigt wird, durchgeführt. Dies änderte jedoch nichts an den Ergebnissen bezüglich der Parteizugehörigkeit. Dennoch ist das Problem fehlender erklärender Variablen natürlich nie vollständig auszuschließen.

9 Getestet wurden die VIF-Werte der Schätzungen, diese sind mit maximal ca. vier immer deutlich unterhalb des kritischen Wertes von zehn (Wooldridge 2013, S. 98). 


\section{Ergebnisse der qualitativen Analyse}

Leitmotiv der qualitativen Analyse war die Untersuchung der akteursbezogenen Erklärungsfaktoren und hier insbesondere die Erklärungskraft der Konstrukte der Konkordanz- und Konkurrenzdemokratie. ${ }^{10}$ Ausgewählt wurden für die 16 Fallstudien zwei ostdeutsche und zwei westdeutsche Bundesländer, die sich hinsichtlich des erwähnten Kommunalverfassungsindex möglichst stark unterschieden und deshalb eher konkordanz- bzw. konkurrenzdemokratische Muster auf kommunaler Ebene erwarten ließen. In Westdeutschland wurde nach diesen Kriterien das klassische Pärchen Nordrhein-Westfalen (im Folgenden NRW) und BadenWürttemberg ausgewählt, während in Ostdeutschland bei deutlich geringerer Varianz des Kommunalverfassungsindex Sachsen und Brandenburg ausgesucht wurden. Die Kommunen für die Fallstudien in den Bundesländern wurden danach bestimmt, dass sie in ähnliche Gemeindegrößenklassen (weitgehend zwischen 20.000 und 100.000 Einwohner) und ähnliche sozialstrukturelle Cluster fallen, um damit den Einfluss exogener sozioökonomischer Erklärungsvariablen auf die Verschuldung möglichst konstant zu halten. Zudem wurden in jedem Bundesland zwischen den vier ausgewählten Kommunen zwei Faktoren systematisch variiert. Selektiert wurden pro Bundesland jeweils zwei Kommunen mit Bürgermeistern mit und ohne Verwaltungshintergrund, zwischen denen wiederum systematisch die Bürgermeister-Ratsrelation variierte. Insgesamt wurden mehr als 70 halbstandardisierte Experteninterviews geführt.

Ein Blick auf die Untersuchungskommunen zeigt (Tabelle 4), dass annahmegemäß in den konkurrenzdemokratischen Kommunen die Kassenkredite und damit auch die Gesamtverschuldung ansteigt, während die Kassenkredite in den anderen Kommunen kaum aus dem Ruder laufen und die Gesamtverschuldung eher reduziert wird. Dies muss allerdings nicht ausschließlich auf diese unterschiedlichen Akteurskonstellationen zurückgeführt werden. Gerade in NRW haben die Untersuchungskommunen schwierigere Startbedingungen, weil sie bei ähnlich hohen Arbeitslosenquoten wie in Ostdeutschland Zahler und nicht Empfänger des Solidaritätspakts sind. Deshalb war näher zu untersuchen, wie sich konkordanz- und konkurrenzdemokratische Muster auf konkrete Entscheidungssituationen und Sparmaßnahmen auswirken.

Im Ergebnis zeigt sich, dass die Untersuchungskommunen weitgehend den zu erwartenden Mustern folgen. In Baden-Württemberg und in Sachsen sind alle acht Kommunen eindeutig konkordanzdemokratisch geprägt, während in NRW alle Kommunen zur Konkurrenzdemokratie tendieren. Die brandenburgischen Kommunen rangieren, wie beim Kommunalverfassungsindex, eher in der Mitte mit zwei eher konkurrenz- und zwei konkordanzdemokratischen Kommunen.

10 Politik und Verwaltung schlagen empirisch in der kommunalen Haushaltkrise sehr unterschiedliche Pfade ein, was neben variierenden institutionellen Rahmenbedingungen vorwiegend auf die Wahrnehmungen und Präferenzen der handelnden Akteure zurückzuführen ist. Haushaltskonsolidierung ist und muss dabei nicht das Maß aller Dinge sein, sondern wird legitimer Weise mit anderen Zielen von Politik und Verwaltung (Effektivität, demokratische Legitimation, Wiederwahl etc.) abgewogen. Bei den hier vorgestellten Analysen wird jedoch (normativ) unterstellt, dass es sinnvoll ist, Haushaltskonsolidierungsmaßnahmen vorzunehmen. 
In den konkordanzdemokratischen Kommunen zeigt sich, dass die Bürgermeister und Kämmerer eine meist herausgehobene, von den meisten Ratsmitgliedern geschätzte Position haben und durch frühzeitige und z. T. harte Eingriffe auf erste Anzeichen einer Haushaltskrise reagieren und damit Kassenkredite und Gesamtverschuldung zurückführen, was sie häufig auch als ihre Aufgabe mit höchster Priorität wahrnehmen. Dies wird im Kern von den meisten Ratsmitgliedern akzeptiert, wobei meist alle Fraktionen in Haushaltskommissionen einbezogen werden und Haushalte nicht selten einstimmig verabschiedet werden. So ist es möglich, dass z. T. transparente Verfahren zur Beteiligung der Kommunalvertretung im Haushaltsprozess etabliert werden, die alle Fraktionen akzeptieren. Eine Trennung zwischen Oppositions- und Mehrheitsfraktionen ist nicht auszumachen und auch der Fraktionszwang ist weniger ausgeprägt. Bürgermeister und Kämmerer können ihre eigenen Mehrheiten für die Konsolidierungspolitik formen, und es drohen kaum Blockaden durch gegenläufige Mehrheiten in der Kommunalvertretung, Mobilisierung der Öffentlichkeit oder Nutzung von direktdemokratischen Vetopositionen durch Parteien.

In Sachsen und Baden-Württemberg ist es wegen des Ausbleibens von „wilden“ Spardebatten außerhalb der dafür vorgesehenen Gremien möglich, den kommunalen Burgfrieden zu wahren. Die aufgrund ihrer institutionellen Position als stark einzuschätzenden Bürgermeister in diesen beiden Ländern befürworten den Konsolidierungskurs und unterstützen ihre Kämmereien und Finanzverantwortlichen, wodurch sich letztlich in der politischen Ratsarbeit kein Widerstand bildet. Im Einzelnen können im Rahmen dieses Beitrags nicht alle Fallkonstellationen in den 16 Kommunen beschrieben werden, aber beispielhaft sollen einige dieser Kommunen vorgestellt werden, um im Vergleich zu den konkurrenzdemokratischen Kommunen die Akteurskonstellationen und Konsolidierungsmaßnahmen veranschaulichen zu können. 
Tabelle 4: Untersuchungskommunen im Überblick

\begin{tabular}{|c|c|c|c|c|c|c|}
\hline & $\begin{array}{c}\text { Kon- } \\
\text { kordanz }\end{array}$ & $\begin{array}{c}\text { Kon- } \\
\text { kurrenz }\end{array}$ & $\begin{array}{c}\text { Kassen- } \\
\text { kredite pro } \\
\text { Einwohner } \\
2004 \\
\text { (gerundet) }\end{array}$ & $\begin{array}{c}\text { Kassen- } \\
\text { kredite pro } \\
\text { Einwohner } \\
2012 \\
\text { (gerundet) }\end{array}$ & \begin{tabular}{|c|} 
Schulden \\
Kernhaushalt \\
(fundierte \\
Schulden \\
und Kassen- \\
kredite) \\
2006 \\
\end{tabular} & \begin{tabular}{|c} 
Schulden \\
Kernhaushalt \\
(fundierte \\
Schulden \\
und Kassen- \\
kredite) \\
2012 \\
\end{tabular} \\
\hline Brandenburg & & & & & & \\
\hline Stadt A & $\mathrm{X}$ & & 448 & 0 & 206 & 219 \\
\hline Stadt B & & $\mathrm{X}$ & 671 & 2102 & 1642 & 2520 \\
\hline Stadt C & $\mathrm{X}$ & & 212 & 0 & 826 & 682 \\
\hline Stadt D & & $\mathrm{X}$ & 1302 & 2236 & 2326 & 2582 \\
\hline Nordrhein-Westfalen & & & & & & \\
\hline Stadt E & & $\mathrm{X}$ & 331 & 2771 & 1744 & 3753 \\
\hline Stadt F & & $\mathrm{X}$ & 0 & 835 & 1765 & 1283 \\
\hline Stadt G & & $\mathrm{X}$ & 982 & 2049 & 2880 & 3178 \\
\hline Stadt $\mathrm{H}$ & & $\mathrm{X}$ & 2221 & 5210 & 3624 & 5896 \\
\hline Baden-Württemberg & & & & & & \\
\hline Stadt I & $\mathrm{X}$ & & 0 & 100 & 1539 & 1516 \\
\hline Stadt J & $\mathrm{X}$ & & 0 & 0 & 186 & 50 \\
\hline Stadt K & $\mathrm{X}$ & & 0 & 0 & 928 & 1.370 \\
\hline Stadt L & $\mathrm{X}$ & & 0 & 0 & 528 & 432 \\
\hline Sachsen & & & & & & \\
\hline Stadt M & $\mathrm{X}$ & & 0 & 0 & 135 & 315 \\
\hline Stadt N & $\mathrm{X}$ & & 106 & 0 & 987 & 779 \\
\hline Stadt O & $\mathrm{X}$ & & 0 & 0 & 1267 & 1155 \\
\hline Stadt P & $\mathrm{X}$ & & 0 & 0 & 585 & 286 \\
\hline
\end{tabular}

Quelle: Eigene Auswertungen auf Basis von Interviews und der Aggregatdaten der Kommunen.

In der baden-württembergischen Stadt L setzte ein mächtiger Finanzbürgermeister mit breitem Aufgabenportfolio und Rückendeckung seiner Oberbürgermeisterin ein langfristig angelegtes Entschuldungsprogramm durch. Es gelang die Nettoneuverschuldung auf null zu senken und Schulden im Kernhaushalt abzutragen. Hierzu setzte man in den vergangenen 15 Jahren drei Mal Haushaltsstrukturkommissionen ein, in denen Einsparpotenziale unter Anleitung der Verwaltung gesucht und die Strukturprozesse ausgearbeitet wurden. Trotz „harter Kämpfe“ ist die Notwendigkeit der Sparprogramme jeweils von allen Fraktionen anerkannt und mitgetragen worden. Die Strukturprozesse beinhalteten jeweils (zuletzt 2010) neben der Verschiebung oder Streichung von Investitionen auch die pauschale Vorgabe an alle Fachbereiche, zehn Prozent ihres Personals einzusparen. Bei Haushaltsverabschiedung gelingt es immer mehr als 90 Prozent Zustimmung im Rat zu erreichen.

In der baden-württembergischen Stadt J fand ebenfalls unter einem parteilosen konsolidierungsorientierten Finanzbürgermeister ein über zehn Jahre laufender Paradigmenwechsel in Verwaltungsvorstand, Rat und Bevölkerung hin zu einem 
langfristig nachhaltigen Wirtschaften statt. Durch Beschluss und Einhaltung von Haushaltsgrundsätzen im Rat wurde das strukturelle Defizit abgebaut und die Entschuldung der Stadt als oberste Priorität definiert, was auch vom Oberbürgermeister überwiegend geteilt wird. Tatsächlich wird im Jahr 2014 die Entschuldung des Kernhaushaltes erreicht. Aus der Akteurssicht wird die langfristige Haushaltsplanung des Finanzbürgermeisters als „die Kandare“ angesehen, mit der er jegliche selbständige Ausgabenneigung der Räte im Keim ersticke (Fraktionsvorsitz CDU). Trotz dieser Funktion als „Bremser“ (FV SPD) und graue Eminenz genießt der Finanzbürgermeister großen Respekt bei den Ratsmitgliedern. Mit Hilfe externer Referenten und Szenarientechniken gelang es schon früh, den Räten zu verdeutlichen, dass der Haushalt der Stadt bei der gegebenen Einnahmesituation die gewünschten Investitionen nicht erwirtschaften kann. Die Räte ließen sich fraktionsübergreifend überzeugen. Gemeinsam identifizierte man umfangreiche Streichposten im laufenden Haushalt und konnte so strukturelle Defizite abwenden.

Unter wenig vorteilhaften Rahmenbedingungen gelang es auch in der sächsischen Stadt N durch eine klare Konsolidierungsstrategie den Abbau sämtlicher Kassenkredite sowie eine erfolgreiche Rückführung der weiteren fundierten Schulden zu erreichen. Ein parteiübergreifendes Commitment zur Konsolidierung und Sparsamkeit wird durch starke Bürgermeister und eine fachlich und politisch anerkannte Kämmerei gestützt. Der Oberbürgermeister (seit 2008) sowie der Finanzbürgermeister (seit 2007) sind in der aktuellen Legislaturperiode parteilos. Der Oberbürgermeister wurde von der CDU aufgestellt und von der SPD, der FDP und der Linken bei der Wahl auch offen unterstützt, was in den konkurrenzdemokratischen Städten NRWs undenkbar wäre. Von Wählergemeinschaft bis zur Linken werden konkordanzdemokratische Einstellungen geteilt: „Und wir haben dann also nicht ein Konkurrenzspiel angefangen, links gegen grün und gegen schwarz und so, sondern wie können wir es zusammen machen? “ (FV Linke). Die Reduzierung des Personalbestandes wurde in der Stadt N bereits 1997 initiiert, da zu dem Zeitpunkt bereits die Anzahl der Beschäftigten im Rathaus in Bezug zur Gesamtbevölkerung den vom Freistaat aufgestellten Richtwert überschritt. Durch die frühzeitige Planung konnte die Stellenreduzierung vor allem über das Einstellungssystem sowie über Altersteilzeit geregelt werden. Der Personalabbau in der Stadt gelang so radikal, dass das Mitte der 1990er Jahre geplante und gebaute neue Rathaus, welches ursprünglich Dienstsitz für rund 200 Verwaltungsmitarbeiter sein sollte, heute bei einer Mitarbeiterzahl von ca. 80 Personen deutlich überdimensioniert ist. Teile des Rathauses werden nun durch die öffentliche Bücherei belegt, wodurch indirekt weitere Sparpotenziale generiert wurden.

In der Stadt A, die im Vergleich zu den anderen brandenburgischen Kommunen eher konkordanzdemokratisch geprägt ist, brachte ein starker SPD-Bürgermeister mit einschneidenden Konsolidierungsmaßnahmen den Haushalt auf Gleichgewichtskurs. Er setzte schon früh die Hochzonung von Aufgaben und deren Finanzierung auf den Kreis durch (Jugendamt) und trieb die Privatisierung des Krankenhauses trotz Bedenken und gegen Kritik innerhalb der eigenen Partei voran. In den Haushaltssicherungskonzepten 2004 und 2005 kalkulierte die Stadt durch die „Veräußerung von Gesellschafteranteilen“ mit Einnahmen von 28 Mio. Euro. 
Damit gelang der vollständige Defizitabbau 2006. Dies beruht nicht nur auf dem Privatisierungserlös, sondern auch auf dem Wegfall der städtischen Haftung für die seit 2001 auflaufenden Verluste in Millionenhöhe des Klinikums. Danach konnten in den Jahren 2008-2011 sogar Überschüsse und eine Rücklage erwirtschaftet werden. Mehrheiten werden in der Stadt A fallweise gesucht, wobei die SPD-Fraktion und die Verwaltung die Haushaltsbeschlüsse prägen. „Meine Aufgabe als Fraktionsvorsitzender besteht darin, fünf Stimmen zu suchen, um die Dinge, die wir durchsetzen wollen, dann auch durchsetzen zu können. Das ist mir seit ich das Amt innehabe auch immer gelungen“ (FV SPD). Nicht nur die weitgehende Kooperation der großen Fraktionen, sondern auch die Wählergemeinschaften, die kaum Fraktionsdisziplin aufweisen, erleichtern diese Aufgabe.

In den konkurrenzdemokratischen Städten in NRW und in Brandenburg verläuft die Haushaltspolitik demgegenüber in ganz anderen Bahnen. Mit der ausgeprägten funktionalen Trennung des Kommunalparlaments in Oppositions- und Regierungsfraktionen ist eine fraktionsübergreifende Politik der Haushaltskonsolidierung in keinem der betrachteten Fälle umzusetzen. Bürgermeister und Kämmerer weisen meist eine stärkere personelle Parteipolitisierung auf und werden in der Regel von den Ratsmitgliedern der jeweils anderen Partei nicht als parteiunabhängige Moderatoren akzeptiert. Bei gegenläufigen Ratsmehrheiten kommt es häufiger zu Blockaden in der Haushaltspolitik und auch die Kämmerer haben in der Regel bei allen Mehrheitskonstellationen keine starke Stellung bzw. werden durch Abwahl ausgetauscht, was in Baden-Württemberg bei einer kondordanzdemokratischen Kommunalverfassung rechtlich gar nicht möglich ist. Hierdurch kommt es in der Konkurrenzdemokratie zu einer hohen Fluktuation der Kämmerer, die damit im Gegensatz zu Baden-Württemberg nicht die Möglichkeit haben, Konsolidierungsprogramme über Jahrzehnte zu begleiten. Selbst wenn sich der Bürgermeister auf eine breitere Mehrheit im konkurrenzdemokratischen Rat stützen kann, ist die Konsolidierungsorientierung eher gering, weil befürchtet wird, dass die Opposition sich mit den Konsolidierungsopfern verbündet, um damit bei den nächsten Ratswahlen, Bürgermeisterwahlen oder auch im Bürgerentscheid punkten zu können. Zur Veranschaulichung dieser Problematiken im Konsolidierungsprozess werden im Folgenden auch auf die konkurrenzdemokratischen Kommunen einige Schlaglichter geworfen.

In der nordrhein-westfälischen Stadt E ohne eine stabile Ratsmehrheit kam es aufgrund der starken Parteipolitisierung der Kommunalpolitik zu einer haushaltspolitischen Blockade. CDU und Grüne erklärten ihre Ablehnung des Haushalts 2006 mit den zu geringen Konsolidierungsanstrengungen des Verwaltungsvorstandes und des 2004 neu ins Amt gewählten SPD-Bürgermeisters. Der abgelehnte Haushaltsentwurf enthielt aber auch eine Grundsteuererhöhung zur Haushaltskonsolidierung, die im Rat nicht mitgetragen wurde. Nach der Haushaltsblockade durch den Rat wurde eine Haushaltsstrukturkommission eingerichtet, um die Fraktionen im Rat stärker einzubinden. In der Haushaltsstrukturkommission kam es erneut zu harten politischen und persönlichen Auseinandersetzungen zwischen den Vertretern der unterschiedlichen Ratsfraktionen. So beklagte der CDU-Fraktionsvorsitzende, dass bei den Konflikten in der Haushaltsstrukturkommission um Personaleinsatz und Organisationsänderungen Vertreter der 
CDU-Fraktion von Ratsmitgliedern anderer Fraktionen als „Kaputtsparer“ und „Arbeitsplatzvernichter“ bezeichnet wurden (Haushaltsrede 2006 FV CDU).

Auch in der nordrhein-westfälischen Stadt F kann sich der SPD-Bürgermeister kaum gegenüber dem von der CDU dominierten Rat durchsetzen. In den letzten Jahren scheiterten mehrere Konsolidierungsinitiativen der Verwaltung, weil sich für geplante Konsolidierungsmaßnahmen nicht die erforderliche Ratsmehrheit fand. Schon im Jahr 2003, noch unter einer absoluten Ratsmehrheit der CDU, verhinderten massive Bürgerproteste die geplante Schließung eines Freibades. Anschließend beschloss der Rat sogar den Ausbau zum „Naturbad“. In den letzten Jahren gelang es organisierten Ortsteilinteressen bei unklaren Mehrheitsverhältnissen im Rat immer wieder geplante Konsolidierungsmaßnahmen durch Proteste zu verhindern. Die Gemeindeprüfungsanstalt stellt in ihrer überörtlichen Prüfung ein quantitativ überdurchschnittliches Schulangebot mit einem hohen Sanierungsbedarf sowie einen hohen Flächenverbrauch pro Schüler fest. Aufgrund dessen und wegen „der extrem sinkenden Schülerzahlen bis zum Jahr 2020“ empfahl die GPA entgegen der bisherigen Praxis der Stadt Schulstandorte zusammenzulegen. Nach der Kommunalwahl 2009 unternahm die Verwaltung unter dem SPD-Bürgermeister mehrere Initiativen zur Schließung von Schulstandorten, für die letztlich nicht die erforderliche Ratsmehrheit zustande kam. Nach anfänglicher Zustimmung vollzogen CDU und FDP bei zwei geplanten Schulschließungen eine politische Kehrtwende, nachdem Bürgerinitiativen protestierten und von anderen Fraktionen im Rat unterstützt wurden. Weil sich die Grünen ohnehin gegen die Schulschließungen positionierten, konnte der Bürgermeister dieses Vorhaben nicht mehr durchsetzen, zumal ihm zusätzlich eine Abwahlinitiative angedroht wurde.

Noch schwerwiegender waren die Konflikte in der nordrhein-westfälischen Stadt G. Unter der parteilosen Bürgermeisterin (die zuvor CDU-Mitglied war) ohne Machtbasis im Rat bestand eine Blockadesituation zwischen den beiden großen Fraktionen und der Bürgermeisterin, die die Fraktionen im Rat aufgrund der ausgeprägten Fraktionsdisziplin auch durchhalten konnten. „Sie werden nicht erleben, dass die SPD-Fraktion nicht vollzählig in den Rat kommt. Sie werden nicht erleben, dass die SPD-Fraktion gemischt abstimmt“ (FV SPD). Der parteigebundene Kämmerer konnte keine Führungsrolle in der Konsolidierung übernehmen, auch weil die Bürgermeisterin nicht mit ihm kooperierte und die Fraktionen nur wenig Interesse an einer Konsolidierungspolitik hatten. Die Grabenkämpfe zwischen Rat und Verwaltungsspitze, zwischen den Parteien und innerhalb der Verwaltungsspitze sorgten dafür, dass es dem Rat im gesamten Untersuchungszeitraum nicht gelang, den Haushalt rechtzeitig zu beschließen. Nachdem in den Haushaltsberatungen im Frühjahr 2007 deutlich geworden war, dass die Ansichten der beiden großen Fraktionen und der Bürgermeisterin zur Haushaltsplanung nicht kompatibel waren, setzte die Kommunalaufsicht einen beratenden Sparkommissar ein. Das Verhältnis zwischen Bürgermeisterin und Rat blieb weiter massiv gestört, und die Beratungsresistenz der Bürgermeisterin führte schließlich zur vorzeitigen Demission des Sparkommissars. Dabei verfügt die Stadt über ganz erhebliches endogenes Konsolidierungspotenzial, das aber wegen Blockaden und geringer Konsolidierungsorientierung nicht ansatzweise ausgeschöpft wird. Die 
aufwändige Infrastruktur (z. B. das große Rathaus, Theater, Museum, Bibliothek) verursacht hohe Unterhaltungskosten bei stetig sinkenden Einwohnerzahlen.

Auch in der nordrhein-westfälischen Stadt $\mathrm{H}$ war die Entscheidungsfähigkeit des Rates in der ersten Wahlperiode ab 2004 durch unklare Mehrheiten gehemmt. Die SPD-Oberbürgermeisterin stand der CDU als der stärksten Fraktion gegenüber. In der stark parteipolitisierten Verwaltungsführung funktionierte die $\mathrm{Zu}$ sammenarbeit zwischen der Oberbürgermeisterin und dem CDU-Kämmerer und seiner Nachfolgerin kaum. Sogenannte „Haushaltsbegleitbeschlüsse“, in denen sich die Fraktionen zur Zusammenarbeit bei der Haushaltskonsolidierung verpflichten, wurden zwar öffentlich wirksam gefasst, eine Umsetzung unterblieb jedoch nach Einschätzung der Bezirksregierung. Die Einigkeit des Rates in Fragen der Konsolidierung stieß immer wieder dort an ihre Grenzen, wo mobilisierungsfähige Interessengruppen betroffen waren und teilweise von der Opposition, wie im Fall der Orchesterliebhaber, auch bewusst aktiviert wurden. Ebenso zurückgenommen durch den Rat wurde die Anhebung der Beiträge für die Kinderbetreuung in der Kindertagespflege, in Kindergärten und der offenen Ganztagsschule. Die Schließung von Grundschulen war umstritten und wurde nach deutlich wahrnehmbaren Protesten von Schülern und Eltern wieder verworfen. Ebenso nach Protesten einstimmig von der Sparliste gestrichen wurden, wie die Bezirksregierung in ihrer Haushaltsverfügung kritisierte, die Schließung eines Fußballstadions und eines Freibads. Als endogene Ursache der Haushaltssituation wird von vielen Gesprächspartnern auf den zu spät eingeleiteten Personalabbau der Stadtverwaltung hingewiesen. Auch die Gemeindeprüfanstalt bescheinigte der Stadt 2006 im Vergleich mit den kreisfreien Städten überdurchschnittliche Personalausgaben. Die 2009 neu gewählte CDU-Kämmerin fiel krankheitsbedingt schnell wieder aus. „Sie war für uns ein ganz großer Hoffnungsträger" und wollte „Klartext“ in Sachen Personalabbau sprechen, sei aber „eingefangen“ worden (FV CDU). Sie brachte in ihrer ersten Haushaltsrede das Konsolidierungsproblem auf den Punkt: „Ich habe bei meinem Dienstantritt [...] gesagt, dass in dieser Verwaltung noch personelle Luft ist. Ich habe für diese Aussage mächtig Prügel bezogen, extern sowie intern [...]. Die Wahrheit ist, dass wir an unseren Personalkosten ersticken“. So kritisierte die Kämmerin die Möglichkeit der Beigeordneten, sich einen eigenen Referenten auszusuchen und den sogenannten „Oberbürgermeistertag“, der allen städtischen Angestellten einen zusätzlichen freien Tag beschert (Lokalzeitung). Nach einem kurzen Comeback im Jahr 2010 meldete sie sich im März 2011 erneut krank und trat im Oktober 2012 in den Vorruhestand.

Nicht besser erging es ihrem Kollegen in der konkurrenzdemokratischen Stadt $\mathrm{D}$ in Brandenburg. Die vom Finanzbeigeordneten vorgelegten Haushaltsentwürfe, die massive Einsparungen vorsahen, fanden keine Mehrheit im Hauptausschuss. Eine SPD-Landtagsabgeordnete warf dem Finanzbürgermeister öffentlich vor, die Stadt kaputtzumachen. Um das strukturelle Haushaltsdefizit von 45 Millionen Euro zu beseitigen, hatte der Finanzbeigeordnete eine umfangreiche Liste von Konsolidierungsmaßnahmen vorgelegt, die unter anderem eine Erhöhung der Grund- und Gewerbesteuer um 20 Prozent, umfangreiche Streichungen und Reduzierungen von Zuschüssen im Sozial- und Kulturbereich sowie einen Haustarif für die städtischen Angestellten vorsah. Daraufhin wurden im Laufe des Jahres 
2004 zwei Abwahlverfahren gegen den Finanzbeigeordneten im Stadtrat eingeleitet, von denen das zweite erfolgreich war. Nach der Abwahl des parteilosen Finanzbeigeordneten und dem Verzicht der Oberbürgermeisterin auf tiefgreifende Konsolidierungsmaßnahmen konnte die Blockade des Haushaltsprozesses u. a. dadurch aufgelöst werden, dass sich die Oberbürgermeisterin und die drei großen Fraktionen darauf einigten, keine weiteren Kürzungen im Bereich der freiwilligen Leistungen mehr vorzunehmen. Man ist stolz auf die großzügige Infrastruktur der Stadt und sieht trotz aus dem Ruder laufenden Kassenkrediten keinen Grund zum Umsteuern.

In der brandenburgischen Stadt B schließlich dominiert eine für die Konkurrenzdemokratie typische Blockade: Es gibt eine Koalition (CDU, SPD und die Bürgervereinigung) gegen die Bürgermeisterin von den Linken. So verabschiedete der Rat in der Stadt B trotz mehrerer Anläufe keinen Haushalt in 2012. Zwischenzeitlich weigerte sich die Bürgermeisterin gar einen weiteren veränderten Entwurf vorzulegen, und die Haushaltsaufsicht empfahl einen externen Sparberater hinzuzuziehen (ein privates Beratungsunternehmen), um die Blockade aufzulösen. Nicht nur diese Blockaden, sondern auch die geringe Konsolidierungsorientierung der Akteure in der Vergangenheit führten dazu, dass die Kassenkredite immer weiter aus dem Ruder liefen.

Insgesamt zeigt sich, dass, auch wenn in den konkurrenzdemokratischen Kommunen viele Haushalte mit Mehrheit relativ unspektakulär beschlossen wurden, es immer wieder zu Entscheidungsblockaden kam, die durch Koalitionswechsel auch mitten in der Legislaturperiode ausgelöst werden konnten und bei konfliktreichen Konsolidierungsmaßnahmen immer als Damoklesschwert über dem Haushaltsberatungsprozess schwebten. Das mit der Einführung der Direktwahl implementierte präsidentielle System ist insbesondere bei Kohabitationskonstellationen nur schwer mit der Konkurrenzdemokratie vereinbar. Dieses Damoklesschwert von drohenden Entscheidungsblockaden und die mögliche Abwahl von Kämmerern und Bürgermeistern trägt damit häufiger zur Vermeidung eines strikteren Konsolidierungskurses bei. Solche Entscheidungsblockaden sind in konkordanzdemokratischen Städten in keinem der betrachteten Fälle zu konstatieren.

\section{Zusammenfassung}

Der Beitrag liefert mehrere neue Erkenntnisse für die Analyse der Verschuldungssituation auf der kommunalen Ebene. Neben den bisher meist für die Verschuldung verantwortlich gemachten exogenen Faktoren, wie sozioökonomische und demografische Variablen, sind es auch endogene Faktoren, die die Verschuldung - gemessen als akkumulierter Schuldenstand und als jährliches Haushaltsdefizit - antreiben. Ein zweites wichtiges Ergebnis ist, dass die unterschiedlichen quantitativen Untersuchungsmethoden in Teilen zu unterschiedlichen Ergebnissen kommen. Die Querschnittsregressionen und die Panel-Regressionen liefern bei den endogenen politischen Variablen teilweise unterschiedliche Befunde, etwa bei der Stärke der Parteifamilien im Stadtrat.

Generell zeigt sich bei beiden Untersuchungsdesigns, dass exogene Faktoren wie der Anteil der in der Grundsicherung befindlichen Personen, die Bedeutung 
als Arbeitsort, die Lage in West- oder Ostdeutschland sowie der Status als kreisfreie Stadt hoch signifikante Erklärungsfaktoren sind. Verschuldung wird stark durch sozioökonomische Problemlagen angetrieben. Hinzu kommen Einnahmegrößen wie das Gewerbesteueraufkommen (für das Verschuldungsniveau) sowie die Höhe der Personalausgaben, die durchgängig signifikant sind.

Bei den endogenen Größen sind vor allem die Größe des Kommunalparlaments und die Ausgestaltung der Kommunalverfassung besonders erklärungskräftig. Die Größe des Parlaments ist zwar einerseits ein Proxy für die Gemeindegröße, aber andererseits auch ein Indikator für eine Pork Barrel-Politik. Die Signifikanz des Kommunalverfassungsindex bestätigt die Banner-These $(1987,2006)$, wonach die institutionalisierte Stärke und die Gestaltungsmacht des Bürgermeisters entscheidend für die Performanz der öffentlichen Finanzen sind.

Ein weiterer wichtiger Befund kann aus der Triangulation der Methoden abgeleitet werden. Dass die Stärke der Parteien in den Kommunalparlamenten keine Rolle spielt, wird durch die qualitative Untersuchung bestätigt. Es zeigt sich vielmehr, dass der Bürgermeister als entscheidender Steuerungsakteur relevanter ist. Zudem ist ein zentrales Ergebnis, dass lokale Konkordanzpolitik sehr viel bessere Ergebnisse hervorbringt als ein konfliktiver Parteienwettbewerb. Konkordanzmechanismen waren dabei in der Lage, Blockadesituationen zu beseitigen. Ferner konnte mit den qualitativen Ergebnissen gezeigt werden, dass der Kommunalaufsicht besondere Bedeutung zukommt. Dieser Faktor, für den im Bundesländervergleich bisher keine empirischen Daten vorliegen, könnte für einen Teil der nicht erklärten Varianz in den quantitativen Modellen verantwortlich sein.

\section{Literatur}

Banner, Gerhard. 1984. Kommunale Steuerung zwischen Gemeindeordnung und Parteipolitik. Die öffentliche Verwaltung 37: 364-372.

Banner, Gerhard. 1987. Haushaltssteuerung und Haushaltskonsolidierung auf kommunaler Ebene - Ein politisches Problem. Zeitschrift für Kommunalfinanzen 87: 50-56.

Banner, Gerhard. 2006. Führung und Leistung der Kommune. Deutsche Zeitschrift für Kommunalwissenschaften 6: 57-69.

Barro, Robert J. 1979. On the Determination of the Public Debt. Journal of Political Economy 87: 940-971.

Boettcher, Florian. 2013. Ursachen kommunaler Haushaltsdefizite. Eine finanz- und politikwissenschaftliche Untersuchung am Beispiel der nordrhein-westfälischen Gemeinden. Berlin: Lit Verlag.

Bogumil, Jörg. 2001. Modernisierung lokaler Politik: kommunale Entscheidungsprozesse im Spannungsfeld zwischen Parteienwettbewerb, Verhandlungszwängen und Ökonomisierung. Baden-Baden: Nomos Verlag.

Bogumil, Jörg, und Lars Holtkamp. 2013. Kommunalpolitik und Kommunalverwaltung: eine praxisorientierte Einführung. Bonn: BpB.

Brender, Adi, und Allan Drazen. 2005. Political budget cycles in new versus established democracies. Journal of Monetary Economics 52: 1271-1295.

Buchanan, James M., und Richard E. Wagner. 1977. Democracy in Deficit: The Political Legacy of Lord Keynes. New York: Academic Press. 
Flick, Uwe. 2008. Triangulation: Eine Einführung. Wiesbaden: VS Verlag für Sozialwissenschaften.

Frankenberg, Dominik, und Martin Junkernheinrich. 2014. Kommunalfinanzen im Jahre 2013. Der lange Weg zur Konsolidierung. In Jahrbuch für öffentliche Finanzen 2014, Hrsg. Martin Junkernheinrich, Stefan Korioth, Thomas Lenk, Henrik Scheller und Matthias Woisin, 299-321. Berlin: BWV.

Greene, William H. 2008. Econometric analysis. Upper Saddle River: Pearson Prentice Hall.

Heinemann, Friedrich, Lars P. Feld, Benny Geis, Christoph Gröpl, Sebastian Hauptmeier, und Alexander Kalb 2009. Der kommunale Kassenkredit zwischen Liquiditätssicherung und Missbrauchsgefahr. Baden-Baden: Nomos Verlag.

Hibbs, Douglas A. 1977. Political Parties and Macroeconomic Policy. American Political Science Review 71: 1467-1487.

Holtkamp, Lars. 2010. Kommunale Haushaltspolitik bei leeren Kassen: Bestandsaufnabme, Konsolidierungsstrategien, Handlungsoptionen. Berlin: Edition Sigma.

Holtkamp, Lars, und Thomas Bathge. 2012a. Bürgerhaushalte auch bei leeren Kassen. Demokratische Gemeinde 2012: 11.

Holtkamp, Lars, und Thomas Bathge. 2012b. Lokale Bürgerbeteiligung in der Haushaltskrise. Der moderne Staat 5: 47-64.

Junkernheinrich, Martin. 2009. Die fiskalische Einbindung der Kommunen in den deutschen Bundesländern. In Jahrbuch für öffentliche Finanzen 2009, Hrsg. Martin Junkernheinrich, Stefan Korioth, Thomas Lenk, Henrik Scheller und Matthias Woisin, 195-220. Berlin: BWV.

Junkernheinrich, Martin. 2012. Kommunale Sozialleistungen im Ländervergleich. Ein Beitrag zur finanzpolitischen Berichterstattung. Zeitschrift für Staats- und Europawissenschaften 2: 196-218.

Junkernheinrich, Martin, Thomas Lenk, Florian Boettcher, Mario Hesse, Benjamin Holler, und Gerhard Micosatt. 2014. Haushaltsausgleich und Schuldenabbau: Konzept zur Rückgewinnung kommunaler Finanzautonomie im Land Nordrhein-Westfalen. Berlin: Analytica Verlag.

Junkernheinrich, Martin, und Gerhard Micosatt. 2008. Kommunaler Finanz- und Schuldenreport Deutschland 2008. Ein Ländervergleich. Gütersloh: Bertelsmann Stiftung.

Junkernheinrich, Martin, und Gerhard Micosatt. 2009. Kommunalstrukturen in Deutschland. Eine Analyse zur länderübergreifenden Vergleichbarkeit kommunaler Finanzkennzablen. Gütersloh: Bertelsmann Stiftung.

Niskanen, William A. 1971. Bureaucracy and Representative Government. Chicago/New York: Aldine Atherton.

Nordhaus, William D. 1975. The Political Business Cycle. Review of Economic Studies 42: 169-190.

Peacock, Alan T., und Jack Wiseman. 1967. The growth of public expenditure in the United Kingdom. London: Allen \& Unwin.

Petersen, M. A. 2008. Estimating Standard Errors in Finance Panel Data Sets: Comparing Approaches. Review of Financial Studies 22: 435-480.

Rose, Richard, und Terence Karran. 1987. Taxation by Political Inertia. Financing the Growth of Government in Britain. London: Allen \& Unwin. 
Schmidt, Manfred G. 1993. Theorien der international vergleichenden Staatstätigkeitsforschung. In Policy-Analyse. Kritik und Neuorientierung (PVS-Sonderheft 24), Hrsg. Adrienne Héritier, 371-393. Opladen: Westdeutscher Verlag.

Schmidt, Manfred G. 2001. Ursachen und Folgen wohlfahrtsstaatlicher Politik. Ein internationaler Vergleich. In Wohlfahrtsstaatliche Politik. Institutionen, politischer Prozess und Leistungsprofil, Hrsg. Manfred G. Schmidt, 33-53. Opladen: Leske + Budrich.

Schulenburg, Klaus. 1999. Direktwahl und kommunalpolitische Führung: Der Übergang zur neuen Gemeindeordnung in Nordrhein-Westfalen. Basel: Leske + Budrich.

Seuberlich, Marc. 2012. Interkommunale Zusammenarbeit als Rettungsanker? - Wie die Länder dieses Instrument für ihre Kommunen nutzen. Der moderne Staat 5: 105-124.

Seuberlich, Marc. 2013. Haushaltskonsolidierung trotz widriger Umstände. In Städte in Not. Wege aus der Schuldenfalle?, Hrsg. Bertelsmann Stiftung, 263-287. Gütersloh: Bertelsmann Stiftung.

Statistisches Bundesamt. 2014a. Integrierte Schulden der Gemeinden und Gemeindeverbände. Wiesbaden.

Statistisches Bundesamt. 2014b. Schulden der öffentlichen Haushalte. Wiesbaden.

Stock, James H., und Mark W. Watson. 2003. Introduction to econometrics. Boston: Addison Wesley.

Timm-Arnold, Klaus-Peter. 2011. Bürgermeister und Parteien in der kommunalen Haushaltspolitik. Endogene Ursachen kommunaler Haushaltsdefizite. Wiesbaden: VS Verlag für Sozialwissenschaften.

Tufte, Edward R. 1978. Political control of the economy. Princeton: Princeton University Press.

Wagschal, Uwe. 1996a. Staatsverschuldung. Ursachen im internationalen Vergleich. Wiesbaden: VS Verlag für Sozialwissenschaften.

Wagschal, Uwe. 1996b. Der Einfluss von Parteien und Wahlen auf die Staatsverschuldung. Swiss Political Science Review 2: 305-328.

Wagschal, Uwe, und Maximilian Grasl. 2014. Kommunale Haushaltspolitik, Ergebnisse einer Befragung der Bürgermeister Deutschlands. Universität Freiburg (Manuskript).

Wagschal, Uwe, Ole Wintermann, und Thiess Petersen (Hrsg.). 2009. Konsolidierungsstrategien der Bundesländer: Verantwortung für die Zukunft. Gütersloh: Bertelsmann Stiftung.

Wooldridge, Jeffrey M. 2013. Introductory Econometrics. A Modern Approach. Mason: South-Western Cengage Learning.

\section{Autorenangaben}

Prof. Dr. Jörg Bogumil,

Ruhr Universität Bochum, Fakultät für Sozialwissenschaft, Lehrstuhl Öffentliche Verwal-

tung, Stadt- und Regionalpolitik,

Universitätsstraße 150, Gebäude GC 05/707,

44801 Bochum,

joerg.bogumil@ruhr-uni-bochum.de 
Prof. Dr. Lars Holtkamp,

FernUniversität Hagen, Lehrgebiet: Politikwissenschaft IV: Politik und Verwaltung,

Universitätsstr. 33,

58084 Hagen,

lars.holtkamp@fernuni-hagen.de

Prof. Dr. Martin Junkernheinrich,

TU Kaiserslautern, Fachbereich Raum- und Umweltplanung, Lehrstuhl Stadt-, Regional-

u. Umweltökonomie,

Pfaffenbergstr. 95,

67663 Kaiserslautern,

martin.junkernheinrich@ru.uni-kl.de

Prof. Dr. Uwe Wagschal,

Albert-Ludwigs-Universität Freiburg, Seminar für Wissenschaftliche Politik, Professur für Vergleichende Regierungslehre,

Werthmannstr. 12,

79098 Freiburg,

uwe.wagschal@politik.uni-freiburg.de

[Korrespondenzautor] 


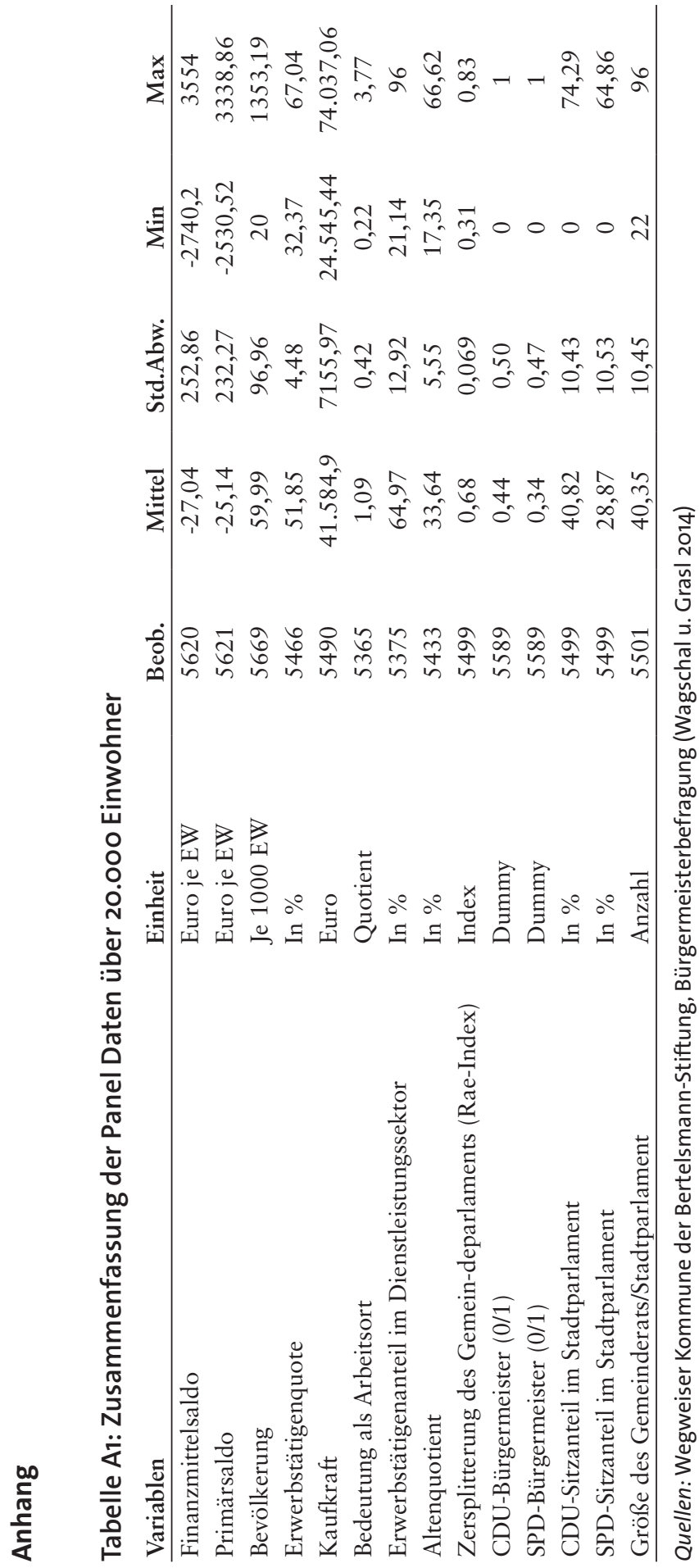

